\title{
AGN variability time scales and the discrete-event model ${ }^{\star}$
}

\author{
P. Favre ${ }^{1,2}$, T. J.-L. Courvoisier ${ }^{1,2}$, and S. Paltani ${ }^{1,3}$ \\ 1 INTEGRAL Science Data Center, 16 Ch. d'Ecogia, 1290 Versoix, Switzerland \\ e-mail: Thierry.Courvoisier@obs.unige.ch \\ 2 Geneva Observatory, 51 Ch. des Maillettes, 1290 Sauverny, Switzerland \\ ${ }^{3}$ Laboratoire d'Astrophysique de Marseille, Traverse du Siphon, BP 8, 13376 Marseille Cedex 12, France
}

Received 25 August 2004 / Accepted 3 August 2005

\section{ABSTRACT}

We analyse the ultraviolet variability time scales in a sample of 15 type 1 Active Galactic Nuclei (AGN) observed by IUE. Using a structure function analysis, we demonstrate the existence in most objects of a maximum variability time scale of the order of $0.02-1.00$ year. We do not find any significant dependence of these maximum variability time scales on the wavelength, but we observe a weak correlation with the average luminosity of the objects. We also observe in several objects the existence of long-term variability, which seems decoupled from the short-term one. We interpret the existence of a maximum variability time scale as a possible evidence that the light curves of type 1 AGN are the result of the superimposition of independent events. In the framework of the so-called discrete-event model, we study the event energy and event rate as a function of the object properties. We confront our results to predictions from existing models based on discrete events. We show that models based on a fixed event energy, like supernova explosions, can be ruled out. In their present form, models based on magnetic blobs are also unable to account for the observed relations. Stellar collision models, while not completely satisfactory, cannot be excluded.

Key words. galaxies: active - galaxies: Seyfert - quasars: general - ultraviolet: galaxies

\section{Introduction}

The UV excess of the spectral energy distribution of type 1 Active Galactic Nuclei (AGN), the blue bump, reflects the fact that a very large fraction of the energy is released in the wavelength domain $\sim 300$ to $5600 \AA$ (see e.g. Krolik 1999, Fig. 7.10). Conventional accretion disk models are able to account satisfactorily for the rough shape of the blue bump (but see Koratkar \& Blaes 1999), but they fail to explain the variability properties (Courvoisier \& Clavel 1991), a key to the understanding of the AGN phenomenon.

This difficulty led several authors (Cid Fernandes et al. 1996; Paltani \& Courvoisier 1997 (hereafter PC97); Aretxaga et al. 1997; Cid Fernandes et al. 2000 (hereafter CSV00); see also Aretxaga \& Terlevich 1994) to consider a more phenomenological approach based on the discrete-event model, which provides a simple explanation for the variability: The variability is the result of a superimposition of independent events occurring at random epochs at a given rate. The motivations for the discrete-event model are twofold: Temporal analysis allows to constrain the event properties, while its generality leaves room for a large variety of physical events. It can be a reasonable approximation for models like starburst (Aretxaga \& Terlevich 1994; Aretxaga et al. 1997), stellar

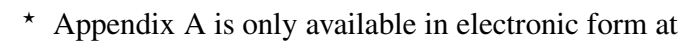
http://www.edpsciences.org collisions (Courvoisier et al. 1996; Torricelli-Ciamponi et al. 2000), or magnetic blobs above an accretion disk (Haardt et al. 1994).

In this paper, we use data from the International Ultraviolet Explorer (IUE) covering about 17 years to determine the UV characteristic time scales in a sample of Seyfert 1 galaxies and QSOs, using a methodology similar to that used in Collier \& Peterson (2001, hereafter CP01). While CP01 concentrated on the measure of time scales shorter than 100 days by selecting short portions of the light curves in which the time sampling was denser, we use here the full available light curves (on average 16.5 years), highlighting a wider range of time scales, and extend the sample to 15 objects. Furthermore, using 12 wavelength windows between 1300 and $3000 \AA$, we investigate for the first time the existence of a wavelength dependence of the variability time scale.

We interpret the variability properties of our objects in terms of discrete-event model, and we study their parameters as a function of the object properties. Our approach is similar to that of CSV00, although we use data from IUE gathered during almost 17 years, while they use optical data covering about seven years. Furthermore, the sample of CSV00 is composed of PG quasars (median $z$ : 0.16) while ours is mainly composed of Seyfert 1 galaxies at a much smaller redshift (median $z$ : 0.033). Their observations thus not only cover about three times less time than ours, but the observation durations are 
further diminished in the observer's frame. Finally, as variability increases towards shorter wavelengths (Kinney et al. 1991; Paltani \& Courvoisier 1994; di Clemente et al. 1996; Trèvese \& Vagnetti 2002), the study of the variability is more efficient in the UV than in the optical.

\section{The concept of discrete-event model}

The formalism of the discrete-event model was mainly developed in Cid Fernandes et al. (1996), PC97, Aretxaga et al. (1997), and CSV00. In the discrete-event model, the variability is due to the superimposition of independent events, occurring at random epochs, on top of a possible constant source. In the simplest form that we use here, all events are identical.

The total luminosity density at wavelength $\lambda$ can be expressed as:

$L_{\lambda}(t)=\sum_{i} e_{\lambda}\left(t-t_{i}\right)+C_{\lambda}$,

where $e_{\lambda}\left(t-t_{i}\right)$ is the light curve of event $i$, initiated at $t_{i}$, and $C_{\lambda}$ reflects the possible contribution of a steady component. The distribution of $t_{i}$ is assumed to be Poissonian. Using $N$ for the event rate and $E_{\lambda}=\int e_{\lambda}\left(t-t_{i}\right) \mathrm{d} t$ for the energy density released by the $i^{\text {th }}$ event, the average luminosity reads:

$\overline{L_{\lambda}}=N E_{\lambda}+C_{\lambda}$.

Parameterizing the event using its duration $2 \mu_{\lambda}$ and its amplitude at maximum $H_{\lambda}$, we have:

$\overline{L_{\lambda}}=N k_{\mathrm{L}} H_{\lambda} 2 \mu_{\lambda}+C_{\lambda}$,

where $k_{\mathrm{L}}$ is a constant depending on the event shape. The variance of $L_{\lambda}(t)$ was calculated by PC97 (see their Eq. (A5)), which reduces to $\operatorname{Var}\left(L_{\lambda}\right) \propto N$. Including $H_{\lambda}$ and $2 \mu_{\lambda}$ in Eq. (B2) of their paper, we find:

$\operatorname{Var}\left(L_{\lambda}\right)=N k_{\mathrm{V}} H_{\lambda}^{2} 2 \mu_{\lambda}$,

where $k_{\mathrm{V}}$ is a constant depending on the event shape. From Eqs. (3) and (4), the event amplitude at wavelength $\lambda$ reads:

$H_{\lambda}=\frac{k_{\mathrm{L}}}{k_{\mathrm{V}}} \frac{\operatorname{Var}\left(L_{\lambda}\right)}{\overline{L_{\lambda}}-C_{\lambda}}$.

In Sect. 4 , we describe a method to estimate $2 \mu_{\lambda}$. As already noted by CSV00, our system will not be closed, as only three parameters can be measured: $\left(\overline{L_{\lambda}}, \operatorname{Var}\left(L_{\lambda}\right), 2 \mu_{\lambda}\right)$, while our model requires the knowledge of six parameters $\left(N, E_{\lambda}, 2 \mu_{\lambda}\right.$, $C_{\lambda}, k_{\mathrm{L}}$, and $k_{\mathrm{V}}$ ). Fixing the event shape determines $k_{\mathrm{L}}$ and $k_{\mathrm{V}} . C_{\lambda}$ is unknown, but constrained in the range $0 \leq C_{\lambda} \leq$ $\min _{t} L_{\lambda}(t)$, see Sect. 5.2. The system can therefore be solved for these limiting cases. Under these assumptions, we can therefore derive the energies and rates of the events from the light curves.

\section{The IUE light curves}

\subsection{Data selection}

We selected all the type 1 AGN spectra available in early December 2001 in the INES (IUE Newly Extracted Spectra)

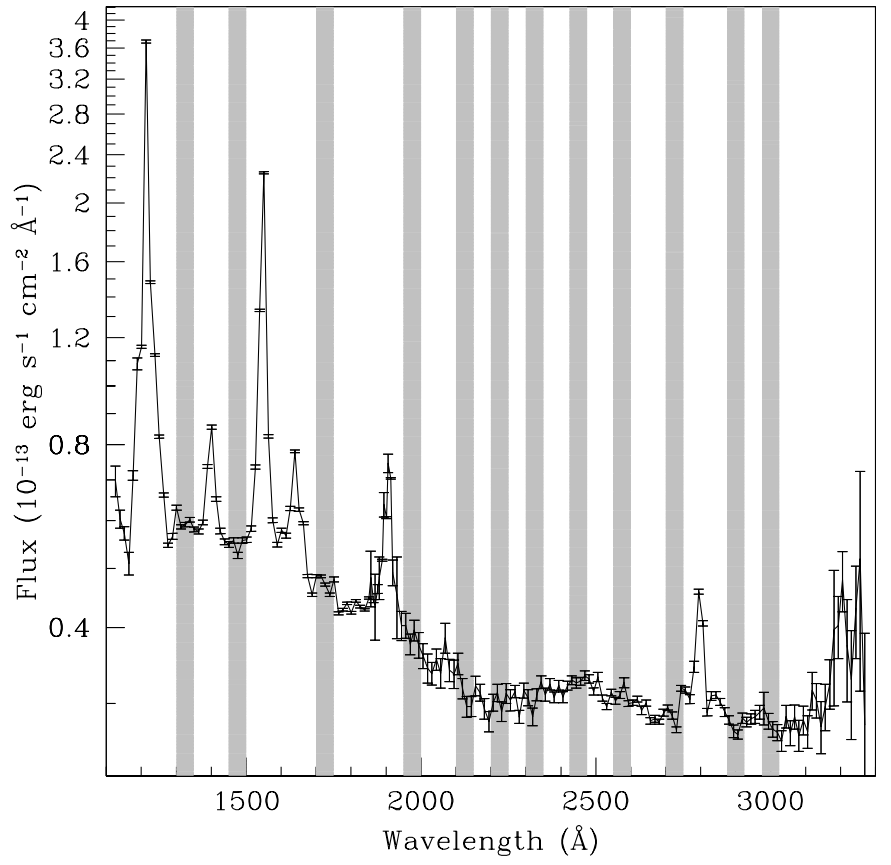

Fig. 1. Average $I U E$ spectrum of the Seyfert 1 galaxy Mrk 335. The position of the 12 spectral windows is indicated by the gray areas.

v3.0 database at VILSPA/LAEFF, which used a new noise model and background determination (Rodríguez-Pascual et al. 1999). We extracted the objects monitored for several years for which at least 20 large aperture, small dispersion observations have been performed with the SWP instrument (1150-1950 ̊). These conditions were imposed by the temporal analysis (see Sect. 4.1). Table 1 gives a list of the selected objects with their common names and redshifts. We finally have in our sample 13 Seyfert 1 galaxies, one broad-line radio galaxy (BLRG), and one quasar.

When building the light curves, FITS headers of all spectra were carefully checked for anomalies. We excluded spectra which were affected by an objective technical problem stated in the FITS headers (e.g., no significant flux detected, object out of aperture, no guiding, no tracking). Spectra for which the pointing direction was farther than $10^{\prime \prime}$ from the object position were also discarded. For 3C 273, the selected spectra correspond to the list described in Türler et al. (1999).

\subsection{Light curves}

For each object, we built 12 light curves in $50 \AA$ spectral windows starting at $1300 \AA, 1450 \AA, 1700 \AA, 1950 \AA, 2100 \AA$, $2200 \AA, 2300 \AA$, $2425 \AA, 2550 \AA, 2700 \AA$, $2875 \AA$, and $2975 \AA$ in the rest frame of the object, avoiding contamination by strong emission lines. Figure 1 shows the average spectrum of Mrk 335, in which the chosen continuum spectral windows have been highlighted.

Two observations showing clearly spurious fluxes were removed; one in NGC 3516 (Julian day: 2449760.78 ), and one in NGC 5548 (Julian day: 2448 993.89). No correction for reddening was applied, as this should have no qualitative influence on our results (See Sect. 5.3). 
Table 1. The 15 objects of the sample. The redshifts are taken from NED. The columns "SWP" and "LWP/R" give the number of observations in each wavelength range. The column " $\Delta T$ " gives the monitoring duration in the rest frame (at $1300 \AA$ ), while the average luminosity $\overline{L_{1300}}$ in the band $1300-1350 \AA$ is given in the seventh column.

\begin{tabular}{lccrrccc}
\hline \hline Name & Classification & $z$ & SWP & LWP/R & $\begin{array}{c}\Delta T \\
(\text { year })\end{array}$ & $\begin{array}{c}\overline{L_{1300}} \\
\left(\times 10^{40} \mathrm{erg} \mathrm{s}^{-1}\right)\end{array}$ & $E_{B-V}$ \\
\hline Mrk 335 & Seyfert 1 & 0.0257 & 26 & 28 & 12.66 & $622.58 \pm 22.78$ & 0.059 \\
Mrk 509 & Seyfert 1 & 0.0343 & 39 & 32 & 15.06 & $1571.8 \pm 67.16$ & 0.060 \\
Mrk 926 & Seyfert 1 & 0.0475 & 22 & 16 & 14.51 & $1182.9 \pm 137.73$ & 0.053 \\
Mrk 1095 & Seyfert 1 & 0.0331 & 35 & 23 & 10.82 & $908.51 \pm 29.44$ & 0.170 \\
NGC 3516 & Seyfert 1 & 0.0088 & 71 & 22 & 16.67 & $40.51 \pm 2.44$ & 0.054 \\
NGC 3783 & Seyfert 1 & 0.0097 & 95 & 84 & 13.54 & $70.01 \pm 2.13$ & 0.141 \\
NGC 4151 & Seyfert 1 & 0.0033 & 153 & 137 & 18.09 & $26.45 \pm 2.17$ & 0.031 \\
NGC 4593 & Seyfert 1 & 0.0083 & 20 & 15 & 8.31 & $12.65 \pm 1.22$ & 0.034 \\
NGC 5548 & Seyfert 1 & 0.0171 & 175 & 148 & 16.60 & $189.70 \pm 5.21$ & 0.024 \\
NGC 7469 & Seyfert 1 & 0.0163 & 65 & 15 & 17.79 & $202.78 \pm 4.59$ & 0.079 \\
3C 120.0 & Seyfert 1 & 0.0330 & 43 & 21 & 15.40 & $159.96 \pm 9.47$ & 0.160 \\
3C 273 & Quasar & 0.1583 & 124 & 114 & 15.36 & $91782 \pm 1610.8$ & 0.027 \\
3C 390.3 & BLRG & 0.0561 & 99 & 11 & 16.37 & $292.98 \pm 16.11$ & 0.071 \\
Fairall 9 & Seyfert 1 & 0.0461 & 139 & 63 & 15.80 & $1981.8 \pm 110.48$ & 0.042 \\
ESO 141-55 & Seyfert 1 & 0.0371 & 26 & 16 & 11.33 & $1785.1 \pm 101.40$ & 0.075 \\
\hline
\end{tabular}

The light curves at $1300-1350 \AA$ for all objects in our sample are presented in Fig. 2. The monitoring durations in the rest frame of the objects are between 8.31 years (NGC 4593) and 18.09 years (NGC 4151), see Table 1.

\section{Temporal analysis}

\subsection{Structure function analysis of the light curves}

The first-order structure function of a light curve $x(t)$ is a function of the time lag $\tau$, and is defined by:

$\mathrm{SF}_{x}(\tau)=\left\langle(x(t+\tau)-x(t))^{2}\right\rangle$,

where $\langle y\rangle$ denotes the average of $y$ over $t$. The structure function (hereafter SF) analysis was introduced in astronomy by Simonetti et al. (1985), and is related to power density spectrum analysis (Paltani 1999). It measures the amount of variability present at a given time scale $\tau$. It has the advantage of working in the time domain, making the method less sensitive to windowing and alias problems than Fourier analysis. We shall use here the property that, if a maximum characteristic time scale $\tau_{\max }$ is present in the light curve, the SF is constant for $\tau \geq \tau_{\max }$, reaching a value equal to twice the variance of $x(t)$. Below $\tau_{\max }$ the SF rises with a logarithmic slope of two at most. On very short time scales, the SF is dominated by the uncertainties on the light curve, and presents a plateau at a value equal to twice the average squared measurement uncertainty.

We estimate the SFs of our light curves by averaging flux differences over predefined time bins, considering only the bins containing at least six pairs. We oversample the SFs (i.e. the bin-to-bin interval is smaller than the bin width) in order to emphasize their characteristics. The bins are geometrically spaced, i.e. the bin-to-bin interval is constant on a Log scale. Finally, we do not attempt to determine error bars on the SFs, as none of the prescriptions found in the literature seem satisfactory. For example, in the prescriptions of
Simonetti et al. (1985) and CP01, the uncertainty on the SF values is proportional to $n_{i}^{-1 / 2}$, where $n_{i}$ is the number of pairs in bin $i$. These prescriptions produce underestimated error bars at large $\tau$ (illustrated in Fig. 3 of CP01), because the number of pairs is increasing roughly exponentially with $\tau$ because of the geometric spacing of the bins, while the total information in the light curve is finite.

Structure functions of the 1300-1350 ̊ light curves for the 15 objects of our sample are presented in Fig. 3. The asymptotic values at twice the light curve variances are also shown. Narrow structures in the SF (see e.g. Fairall 9, 3C 390.3) are very probably due to the high inter-correlation of the SF (because the same measurements are used in several bins), and are probably not physical.

In Fig. 3, we observe that, for a majority of objects, the SFs show a plateau at about twice the variance of the light curves. However, in some cases, the SF continues to increase for $\tau$ larger than $\sim 5$ years. Paltani et al. (1998) presented a similar analysis on 3C 273, and concluded that a second component, varying on long time scales, was present, particularly at long wavelengths. Such a component appears in the form of a SF rising sharply at large $\tau$. To cope with the possibility of the existence of a second component, we fit the SFs using the same function as in Paltani et al. (1998):

$\mathrm{SF}_{x}(\tau)=2 \epsilon^{2}+\left\{\begin{array}{cc}A\left(\tau / \tau_{\max }\right)^{\alpha}, & \tau<\tau_{\max } \\ A, & \tau \geq \tau_{\max }\end{array}\right\}+B \tau^{\beta}$

where $\epsilon$ is the average uncertainty on the light curves, $\tau_{\max }$ the maximum variability time scale measured at the start of the upper plateau. $A$ and $\alpha$ are the parameters of a first component of variability. $B$ and $\beta$ are the parameters of a second component. As we assume that this second component is slowly varying, we adopt $\beta=2$, the maximum value for the slope of an SF, which indicates very slow variability. 


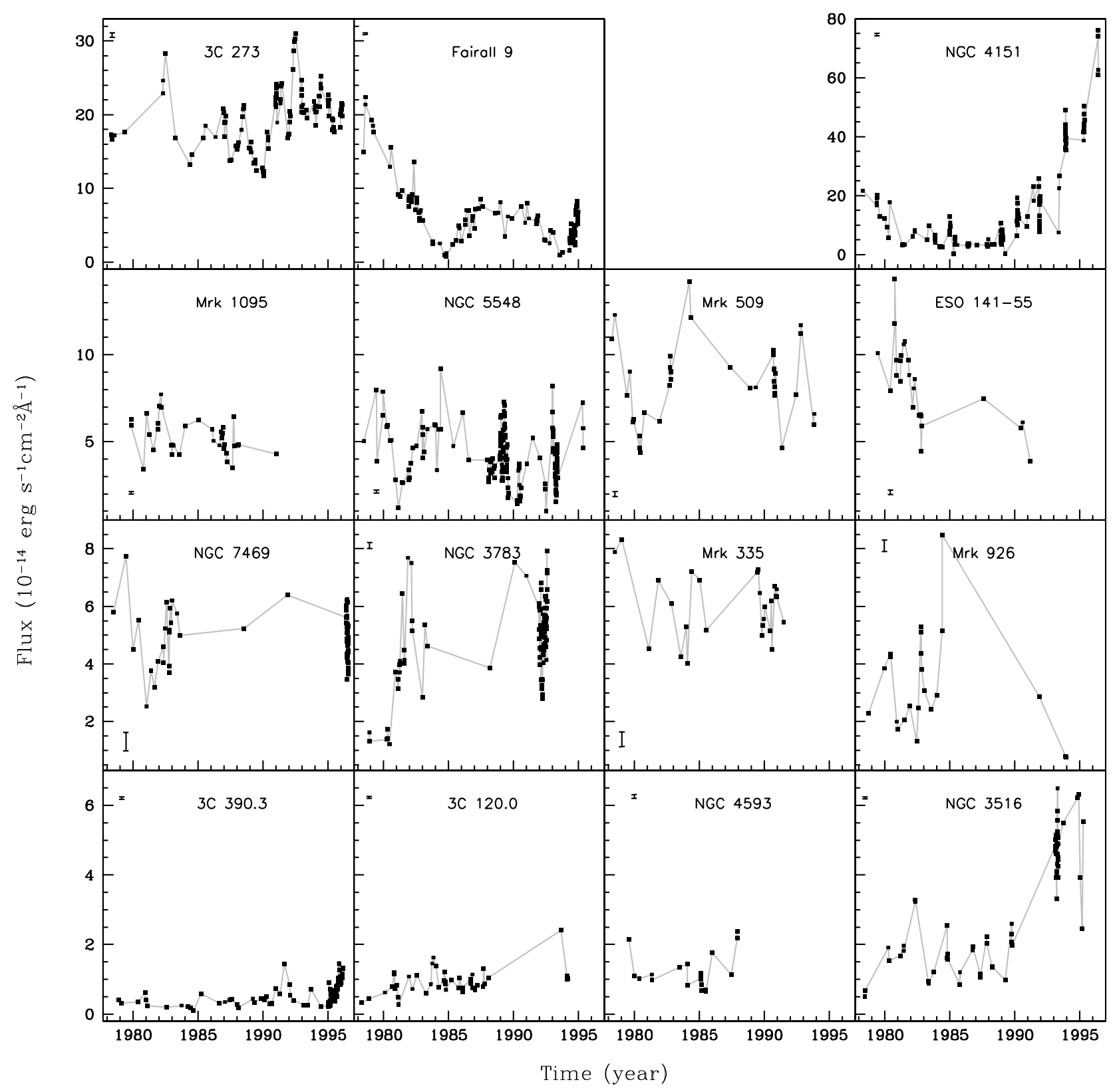

Fig. 2. Light curves in the range 1300-1350 A for the 15 objects of our sample. Only one error bar is drawn on each panel for clarity (in the upper- or lower-left corner).

We first fit the 1300-1350 $\AA$ SFs; we shall discuss the longer wavelength SFs in Sect. 4.3. The values of $\tau_{\max }$ are given in Table 2, corrected for time dilatation. The best fits are shown in Fig. 3 by a continuous line. $\epsilon$ was fitted as a free parameter. The best fit values found for these parameters were comparable to the noise variance mesured in the light curves. In four objects (NGC 3516, NGC 4151, NGC 7469, Fairall 9), the fit does not converge unless we fix the noise parameter $\epsilon$ and the slope $\alpha$ in the fits. We have tested that reasonable choices of $\epsilon$ and $\alpha$ have little influence on the value of $\tau_{\max }$ in those four SFs. We checked as well that the monitoring duration $(\Delta T$ in Table 1) had no influence on the measure of $\tau_{\max }$.

The SFs of NGC 3516, and NGC 4151 show strong structures between 0.1 and 1 year, but their behavior at $\tau>1$ is compatible with an extrapolation of their behavior at $\tau<0.1$.
Our fit interprets the strong structures as evidence of a $\tau_{\max }$, but we need to check that the structures themselves do not result from the sampling. To do that, we simulated light curves using a random walk, and projected them on the original light curve sampling. None of our simulations reproduced the observed structures in the SFs, and we consider therefore that a maximum time scale is really present in these two objects. The shape of the SFs forces us however to make the error bars on $\tau_{\max }$ extend up to 1 year in these two objects. In Fairall 9, no plateau can be seen, but there is a clear change in the slope of the SF between $\tau=0.1$ year and $\tau=3$ years. We interpret this as the presence of a $\tau_{\max }$ in this range of time scale, followed by a very strong slowly varying component. In NGC 3783, a similar change of slope occurs, and it is impossible to locate $\tau_{\max }$ unequivocally. This is reflected in the error bar on $\tau_{\max }$ for 


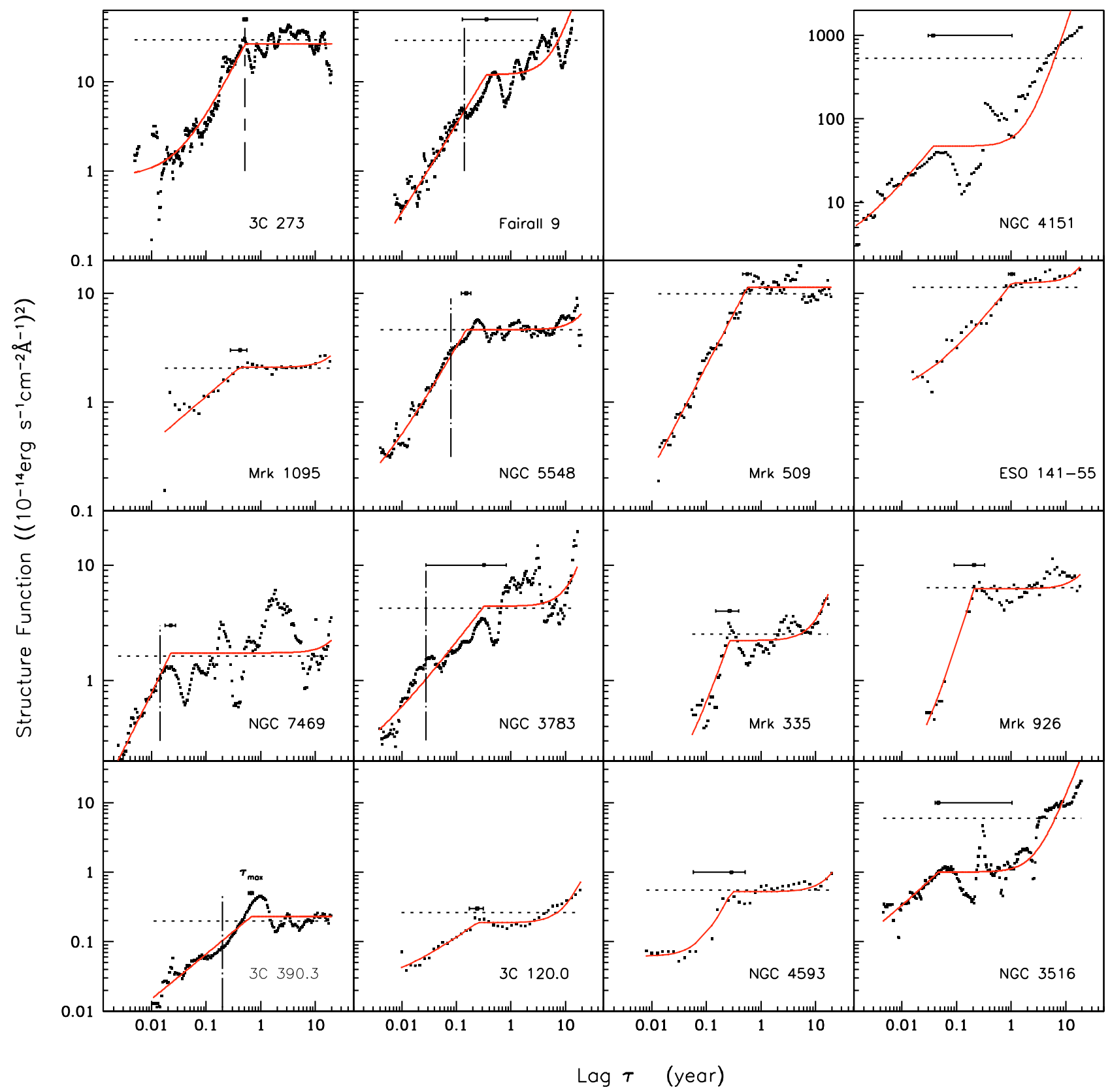

Fig. 3. Structure functions of the light curves at 1300-1350 $\AA$. The horizontal dashed lines show the asymptotic value of twice the variance of $x(t)$. The continuous line shows the best fit, while the value of $\tau_{\max }$ is shown with its uncertainty above the SF to ease readability. The vertical dot-long dash lines indicate the location of the characteristic time scales found in CP01, while the short dash-long dash line indicates the value found in Paltani et al. (1998).

this object. Several other objects show the existence of a second variability component, but it does not affect the measurement of $\tau_{\max }$.

We repeated the analysis of the SFs without including a slow component of variability in the model, i.e. we fitted the data with $B=0$. The values of $\tau_{\max }$ found are compared in Table 2. The values found with $B=0$ are all inside the error bars except for NGC 3516, NGC 3783 and NGC 4151. For NGC 3516 and NGC 4151, the fits do not represent the data. Thus for a majority of objects, the addition of a second component has no effect while it significantly improves the fits for NGC 3516, NGC 3783 and NGC 4151.
We conclude that with these data, we cannot decide if a second component is detected or not. This second variability component, while interesting per se, is outside the scope of this paper, and shall not be discussed further.

For all the objects, we estimate the effect of the binning on $\tau_{\max }$ by computing and fitting 100 SFs (at 1300-1350 ̊) for each object with different binnings, with corresponding bin-tobin intervals between 0.002 year and 0.1 year. For all objects, the distribution of the measured $\tau_{\max }$ is mono-peaked, meaning that a single value of $\tau_{\max }$ was always found by the algorithm. The width of this peak determines an empirical uncertainty on $\tau_{\max }$ (Table 2). 
Table 2. Maximum variability time scales $\tau_{\max }$ of the 1300-1350 light curves, corrected for time dilatation. The third column shows the values of $\tau_{\max }$ obtained with $B=0$, i.e. the SFs have been fitted without a slow variability component. The last column presents the corresponding range of event durations $2 \mu_{1300}$, deduced from the simulations.

\begin{tabular}{lccc}
\hline \hline Object & $\begin{array}{c}\tau_{\max } \\
\text { (year) }\end{array}$ & $\begin{array}{c}\tau_{\text {max }, \mathrm{B}=0} \\
\text { (year) }\end{array}$ & $\begin{array}{c}2 \mu_{1300} \\
\text { (year) }\end{array}$ \\
\hline Mrk 335 & $0.260 \pm 0.120$ & 0.222 & $0.238 \pm 0.169$ \\
Mrk 509 & $0.550 \pm 0.100$ & 0.550 & $0.489 \pm 0.381$ \\
Mrk 926 & $0.200 \pm 0.120$ & 0.227 & $0.180_{-0.120}^{+0.239}$ \\
Mrk 1095 & $0.400 \pm 0.140$ & 0.438 & $0.242_{-0.140}^{+0.371}$ \\
NGC 3516 & $0.046_{-0.006}^{+1.009}$ & 4.896 & $0.050_{-0.010}^{+1.350}$ \\
NGC 3783 & $0.319_{-0.200}^{+0.501}$ & 1.229 & $0.300_{-0.200}^{+1.500}$ \\
NGC 4151 & $0.037_{-0.007}^{+1.003}$ & 2.200 & $0.040_{-0.010}^{+1.500}$ \\
NGC 4593 & $0.284 \pm 0.230$ & 0.262 & $0.686 \pm 0.452$ \\
NGC 5548 & $0.150 \pm 0.030$ & 0.154 & $0.196 \pm 0.063$ \\
NGC 7469 & $0.022 \pm 0.005$ & 0.023 & $0.035 \pm 0.014$ \\
3C 120.0 & $0.234 \pm 0.070$ & 0.287 & $0.321 \pm 0.119$ \\
3C 273 & $0.452 \pm 0.050$ & 0.459 & $0.559 \pm 0.117$ \\
3C 390.3 & $0.631 \pm 0.070$ & 0.635 & $0.498 \pm 0.240$ \\
Fairall 9 & $0.341_{-0.227}^{+2.717}$ & 0.760 & $0.350_{-0.250}^{+2.800}$ \\
ESO 141-55 & $0.997 \pm 0.130$ & 1.014 & $1.630 \pm 0.816$ \\
\hline
\end{tabular}

We measure $\tau_{\max }$ from 0.022 to 0.997 year for the $15 \mathrm{ob}-$ jects of our sample. Our time scales are of the same order of magnitude as the one found by previous studies in the opticalUV (Hook et al. 1994; Trevese et al. 1994; Cristiani et al. 1996; Paltani et al. 1998; Giveon et al. 1999; CSV00). The time scales found by CP01 and Paltani et al. (1998) are indicated in Fig. 3 by a vertical line and are in reasonable agreement with what we have found, 3C 390.3 and NGC 3783 excepted. The discrepancy can be explained by the fact that CP01 "detrend" their SFs with a linear component (i.e. they remove a linear fit from their light curves), arguing that the measured SF will deviate from their theoretical shape if the light curve shows a linear trend. Our method of including a second, slowly variable component in the structure functions is more general than the "detrending", because it makes less strict assumptions on the temporal properties of the slowly varying component. It is nevertheless equivalent in the case where a linear trend is effectively present in the data. Our method is also more consistent in the sense that all components are handled in a similar way. Furthermore, a linear trend would make little sense in objects like NGC 4151.

\subsection{Relation with the event duration}

An easy way to explain the existence of a maximum variability time scale is provided by the discrete-event model. For a Poissonian sequence of events, the SF is proportional to the SF of a single event (Paltani 1996; Aretxaga et al. 1997; CSV00) and only has structures on time scales shorter than the event duration. We interpret the observed SFs using discrete events for which we assume a triangular, symmetric shape. The event shape was chosen for its simplicity (Paltani et al. 1998), but it has been shown that choosing other shapes does not affect

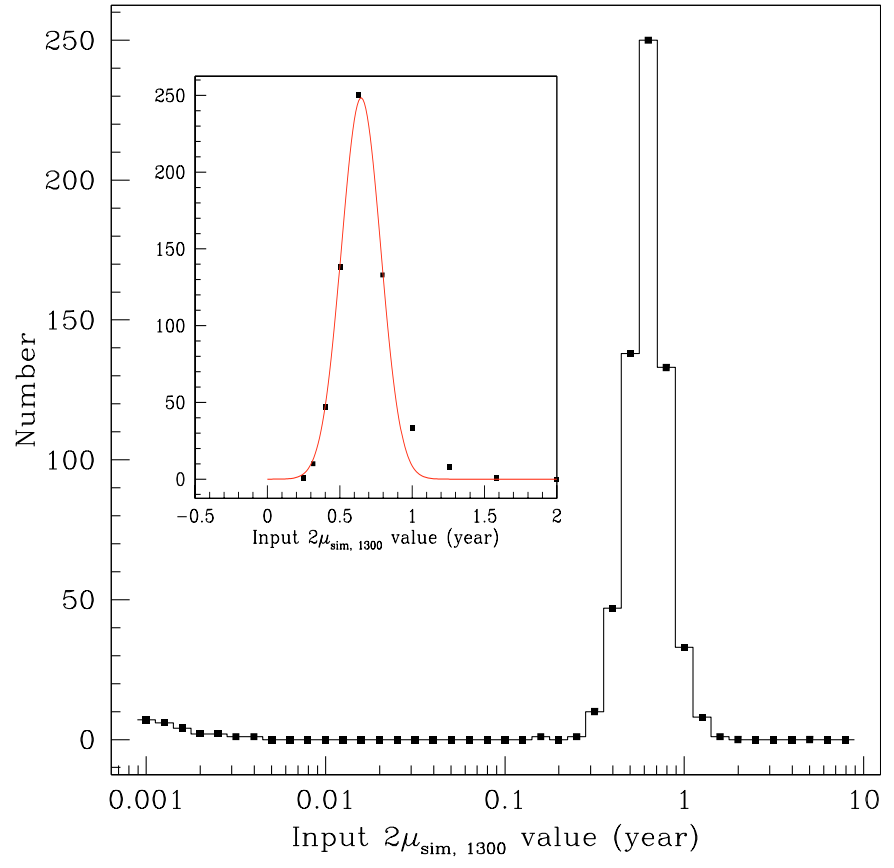

Fig. 4. Histogram of the event durations $2 \mu_{\text {sim, } 1300}$ input to the simulation that produce a measured $\tau_{\max }$ in the range $0.4-0.5$ years, for $3 \mathrm{C} 273$. The inset shows the same diagram in linear scale, fitted with a Gaussian.

significantly the results given by the temporal analysis (CSV00). Our events are described with only two parameters at wavelength $\lambda$ : the event amplitude $H_{\lambda}$ and the event duration $2 \mu_{\lambda} ; \mu_{\lambda}$ being defined as the time needed to reach the maximum flux. It follows that $k_{\mathrm{L}}=1 / 2$ and $k_{\mathrm{V}}=1 / 3$ in this case (see Sect. 2).

While the SFs are in theory able to measure the event duration, this measure can be affected by the noise and sampling of the light curve in a complex and unpredictable way. To test if $\tau_{\max }$ measures a property of the light curves, and not of the sampling, we produce synthetic light curves by simulation, and measure their $\tau_{\max }$. In the simulations, we add randomly triangular events with a given duration $2 \mu_{\text {sim,1300, keeping the same }}$ sampling as the original light curve. A noise with an amplitude equal to the average of the instrumental noise is added to each light curve. We take 40 test values for $2 \mu_{\text {sim, } 1300}$, from $10^{-3}$ to 10 years, geometrically spaced.

For each object and each $2 \mu_{\text {sim,1300, we build } 1000 \text { light }}$ curves, compute their SFs and, measure $\tau_{\max }$ using the method described above. The event rate $N$ is randomly chosen between 5 and 500 events per year. The result of the simulation is, for each object and each $2 \mu_{\text {sim, } 1300}$, the distribution of the resulting $\tau_{\max }$. These distributions allow us to determine which input $2 \mu_{\text {sim, } 1300}$ can provide the observed $\tau_{\max }$. For $3 \mathrm{C} 273$ for example, the distribution of $2 \mu_{\text {sim, } 1300}$ produced a peak around 0.56 year, as represented in Fig. 4 . The fit of the peak of Fig. 4 with a Gaussian gives $2 \mu_{\text {sim, } 1300}=0.559 \pm 0.117$ years.

We note that we never observe a $B$ parameter (see Sect. 4.1) significantly larger than 0 in our simulations. This is expected as we do not include a second component.

The simulations for all objects showed a result qualitatively identical to that for $3 \mathrm{C} 273$, i.e. the distributions of $2 \mu_{\mathrm{sim}, 1300}$ 

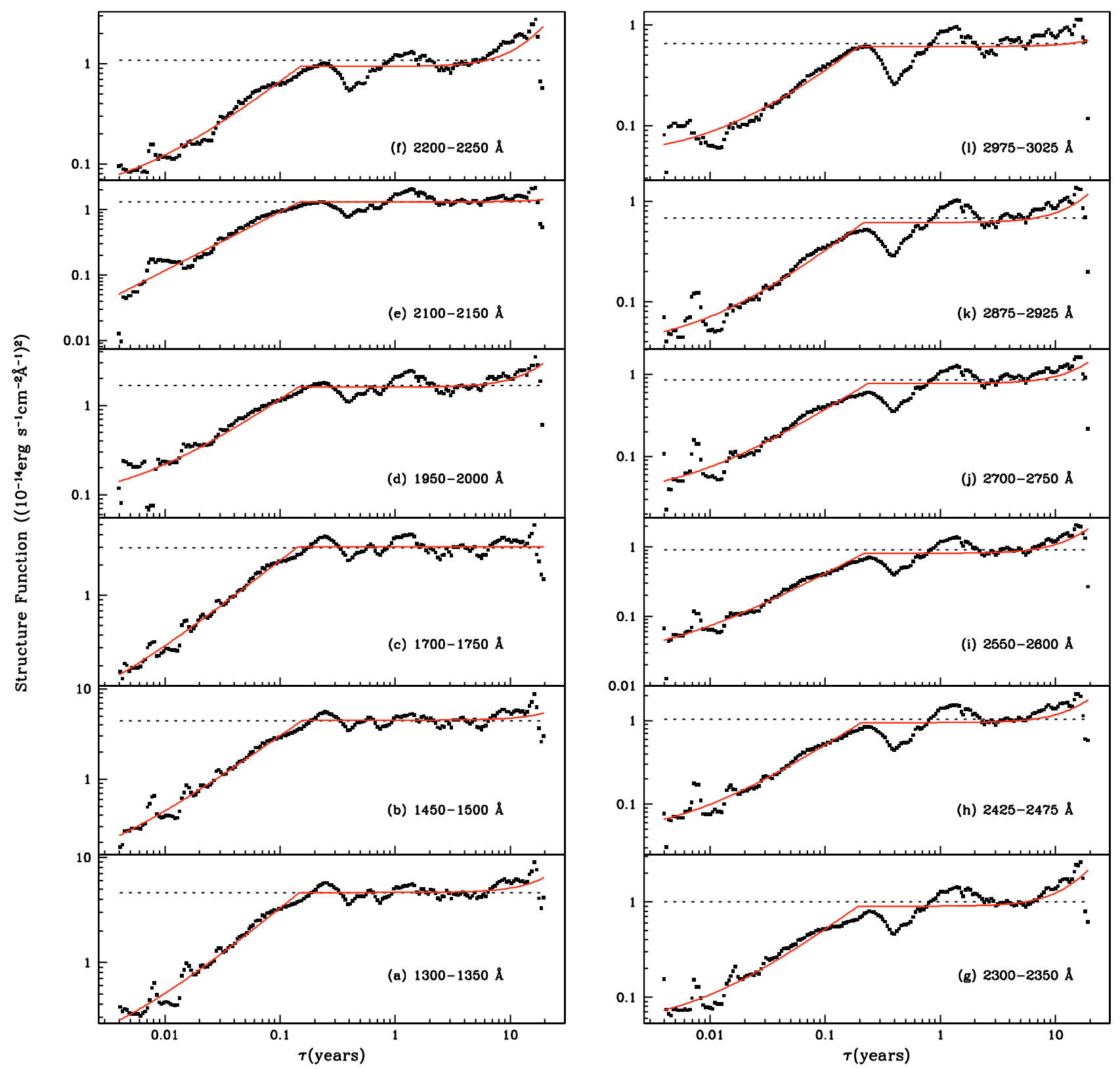

Fig. 5. SFs for the light curves 1300 to $3000 \AA$, for NGC 5548. The horizontal dashed lines show the asymptotic value of twice the variance of the light curve. The best fits are shown by a continuous line.

present a single peak. This means therefore that, for each object, $\tau_{\max }$ determines a unique event duration, that can be derived from the simulations. The values of $2 \mu_{1300}$ are given in Table 2 . We stress that our simulations are driven by the real sampling of the light curves, and are therefore more specific than, for example, those discussed in Welsh (1999), or CP01.

\subsection{Wavelength dependence of the event duration}

For each object of the sample, we apply the method described in Sect. 4.1 to compute the event durations from the light curves 1450 to $2975 \AA$.

Structure functions from $1300 \AA$ to $3000 \AA$ are presented in Appendix A for each object, along with a description of the particularity of each set of SFs. As an example, we show the case of NGC 5548 in Fig. 5.

It is not possible to deduce a value of $\tau_{\max }$ for all 180 light curves. This is mainly due to the fact that, for some of the objects, the number of observations in the LW range is too small. In addition some particular light curves are very noisy. In such cases, the SF usually does not have the canonical shape, and the fit does not succeed. We thus reject the time scale corresponding to those particular SFs. Each individual case is described in Appendix A. In some cases, the noise is such that, although $\tau_{\max }$ can be derived from the SF, its uncertainty is very large. In such cases, one should interpret any variation in $\tau_{\max }$ with caution.

Figure 6 presents the variability time scale $\tau_{\max }$ as a function of the wavelength for all the objects. We find that $\tau_{\max }$ is 


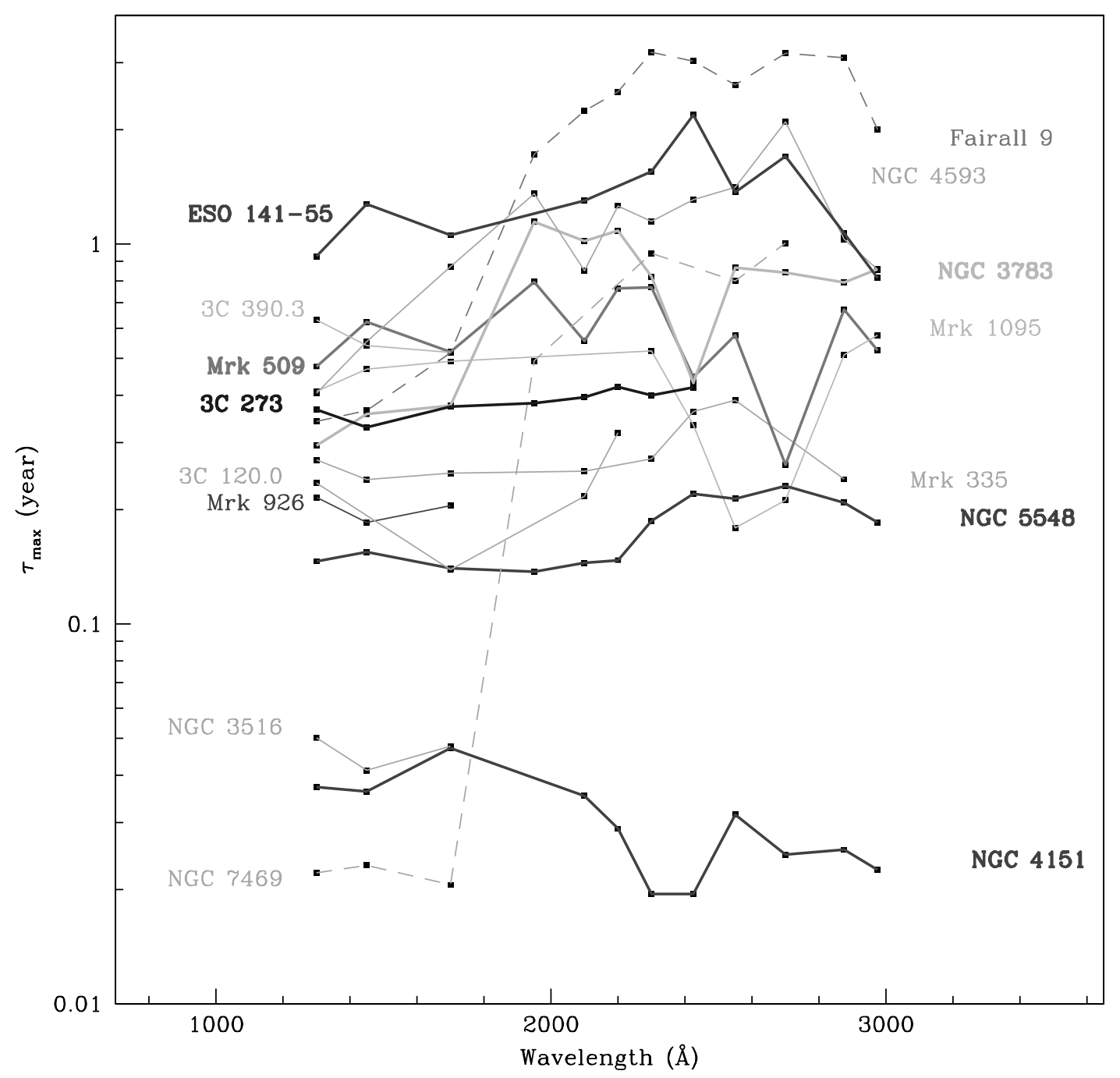

Fig. 6. $\tau_{\max }$ as a function of wavelength for the 15 objects. The $\tau_{\max }$ are corrected for time dilatation. The two objects which present a very strong increase in $\tau_{\max }$ at long wavelengths are shown with a dashed line.

reasonably constant over the wavelength range we use, as the small fluctuations can be explained by the difficulty to measure a precise value of $\tau_{\max }$ on some noisy SF. For the particular case of NGC 5548 for example, the variations are inside the uncertainties derived in the previous section. This result was a also found by Paltani et al. (1998) for 3C 273.

In two objects however, NGC 7469 and Fairall 9, the SFs present a very strong increase in $\tau_{\max }$ at long wavelengths. For NGC 7469, this is due to a lack of short term sampling of the LW light curves, which prevents the recovery of any time scale below 0.5 year. In Fairall 9, a similar lack of short term sampling affects the determination of $\tau_{\max }$. However, the values of $\tau_{\max }$ in the LW range are within the uncertainties on $\tau_{\max }$ determined at $1300 \AA$.

\section{Interpretation in terms of discrete-event model}

\section{1. $2 \mu_{1300}$ as a function of the luminosity}

The relationship between the event duration and the luminosity has some important consequences for the discrete-event model that we will discuss below (Sect. 5.4). We note, however, that only a weak dependence between the event duration and the average luminosity of the object is possible as the values of the former cover less than two orders of magnitude while the latter covers four orders of magnitude. We thus measure a physical time scale which has at most a small dependence on the luminosity of the objects.

The event duration $2 \mu_{1300}$ as a function of the average luminosity $\overline{L_{1300}}$ of the object at $1300-1350 \AA$ is shown in Fig. 7 . We use a simple cosmology with $H_{0}=60 \mathrm{~km} \mathrm{~s}^{-1} \mathrm{Mpc}^{-1}$, and $q_{0}=0.5$ throughout

Using Spearman's correlation coefficient, we find a correlation between the event duration and the luminosity $(s=0.38)$, marginally significant at the $16 \%$ level (Null hypothesis). The dependence of $2 \mu_{1300}$ on $\overline{L_{1300}}$ can be expressed as $2 \mu_{1300} \propto$ $\vec{L}_{1300}$, where the index $\delta=0.21 \pm 0.11$ has been determined using the BCES linear regressions (Akritas \& Bershady 1996).

\subsection{The steady component $C_{\lambda}$}

The steady component $C_{\lambda}$ (Sect. 2) can have various physical origins. For example, it can be associated to the non-flaring 


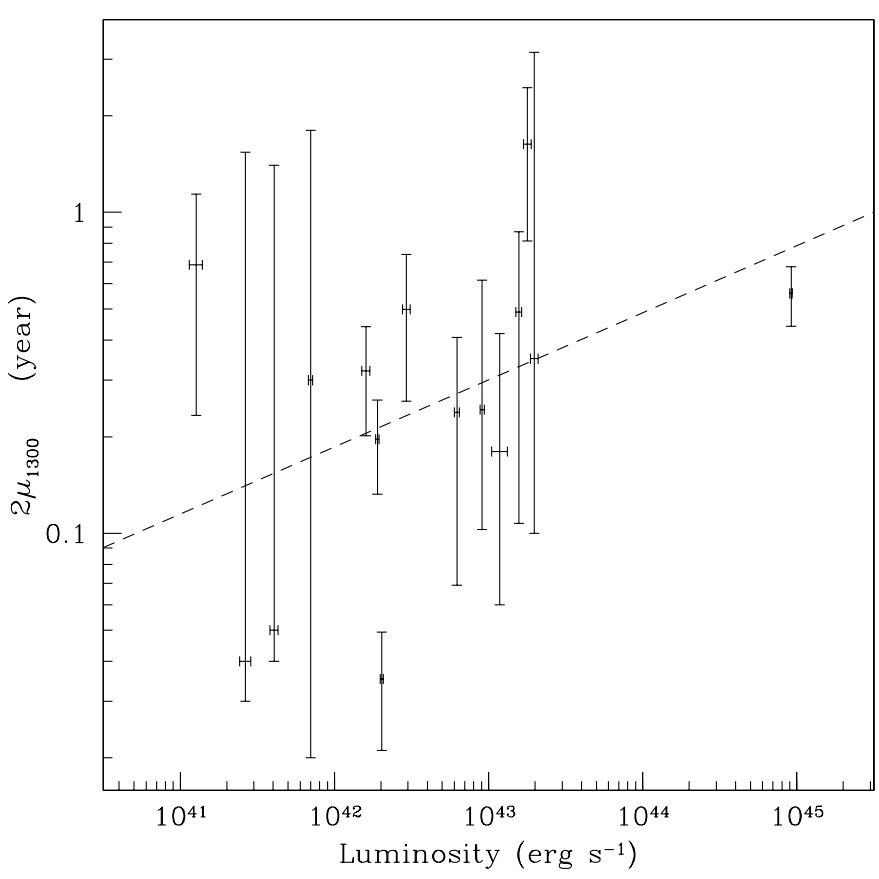

Fig. 7. Variability time scales $2 \mu_{1300}$ as a function of the luminosity of the objects in the range 1300-1350 A. The BCES regression is shown with the dashed line.

part of the accretion disk, or to the host-galaxy stellar contribution (Cid Fernandes et al. 1996; CSV00). We shall however continue our discussion in a model-independent way. We can constrain $C_{\lambda}$ for a particular light curve by imposing that it does not exceed the minimum observed luminosity $L_{\lambda}^{\mathrm{min}}$. On the other hand, $C_{\lambda}=0$ is an obvious lower limit (although Paltani $\&$ Walter (1996) argued that $C_{\lambda}>0$, at least for $\lambda>2000 \AA$ ). In the following, we shall use these two constraints as limiting cases.

\subsection{Spectral shape of the event amplitude, event energy and event rate}

For each object, we compute the event amplitude $H_{\lambda}$ from Eq. (5) using both $C_{\lambda}=0$ and $C_{\lambda}=L_{\lambda}^{\mathrm{min}}$. Figure 8 shows the spectral shape of $H_{\lambda}$ for NGC 5548. We integrate $E_{\lambda}=$ $2 \mu_{\lambda} k_{\mathrm{L}} H_{\lambda}$ interpolated over the wavelength range 1300-3000 to obtain the energy $E$ released in one event, assuming isotropic emission:

$E=\int_{1300 \AA}^{3000 \AA} E_{\lambda} \mathrm{d} \lambda$.

We use the event duration at $1300-1350 \AA$, as it can be considered constant over the wavelength range considered. The event rates $N$ are derived from Eq. (3).

Table 3 gives the event energies and rates found with the $C_{\lambda}=0$, and $C_{\lambda}=L_{\lambda}^{\min }$ assumptions. Event energies are found in the range $10^{48}-10^{52} \mathrm{erg}$, and maximum event rates in the range $9-1133$ event year ${ }^{-1}\left(C_{\lambda}=0\right)$ while minimum event rates are found in the range 2-270 event year ${ }^{-1}$ (upper limit of $C_{\lambda}$ ). Fig. 9 shows the event energy $E$ as a function of the 1300 $1350 \AA$ A luminosity, using the upper and lower limits on $C_{\lambda}$.

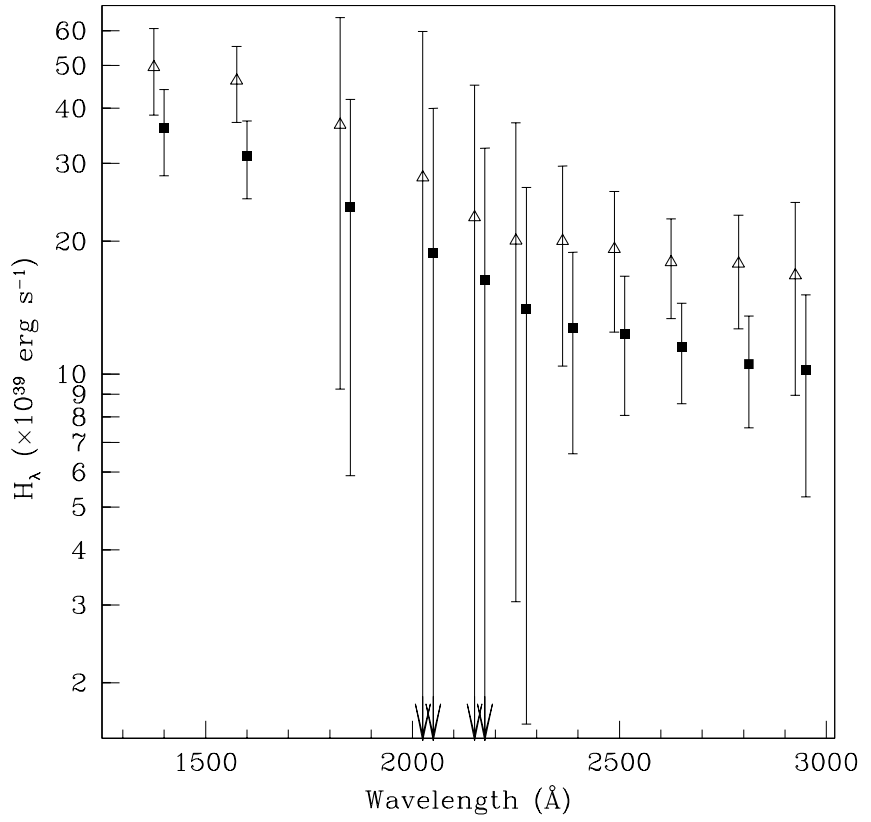

Fig. 8. Event amplitude spectrum $H_{\lambda}$ as a function of wavelength for NGC 5548, with $C_{\lambda}=0$ (squares), and $C_{\lambda}=L_{\lambda}^{\mathrm{min}}$ (open triangles). The lower curve has been slightly shifted to the right to ease readability.

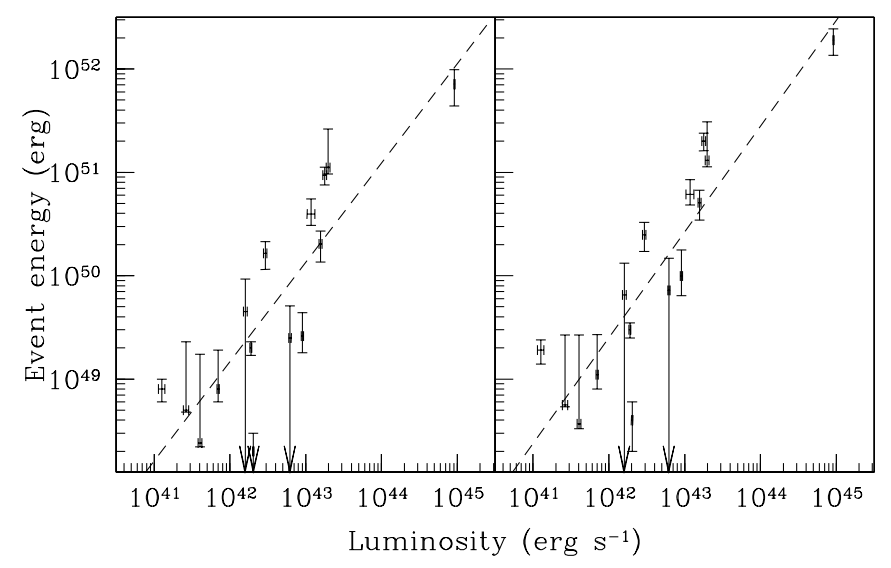

Fig. 9. Event energy as a function of the object luminosity in the hypothesis $C_{\lambda}=0$ (left panel), and using the upper limit of $C_{\lambda}$ (right panel). The BCES regression is shown with the dashed line.

With $C_{\lambda}=0, E$ and $\overline{L_{1300}}$ are clearly correlated (Spearman correlation coefficient: $s=0.85$ ), with a Null hypothesis probability of less than $0.1 \%$. A linear regression gives $E \propto{\overline{L_{1300}}}_{\text {, }}$, with $\gamma=0.96 \pm 0.09$ using the BCES method (Akritas \& Bershady 1996) (Fig. 9, left panel).

When using $C_{\lambda}=L_{\lambda}^{\mathrm{min}}$, the correlation is preserved (Spearman: $s=0.89$ ), with a Null hypothesis probability of less than $0.1 \%$ and an index $\gamma=1.02 \pm 0.11$ (Fig. 9, right panel). We note that $E$ and $\overline{L_{1300}}$ are lower limits since no corrections for reddening were applied. The correlation should be preserved by applying the corrections, as both $E$ and $\overline{L_{1300}}$ would be scaled by the same factor. We tested this hypothesis by correcting the light curves for reddening and recomputing the relation $E=f\left(\overline{L_{1300}}\right)$. The correlation is preserved (Spearman correlation coefficient: $s=0.83$ ) with an index $\gamma=0.93 \pm 0.10$ for the case $C_{\lambda}=0$ as well as for the case $C_{\lambda}=L_{\lambda}^{\text {min }}$ (Spearman 
Table 3. Event rate $N$ and energy $E$ for each object, with the $C_{\lambda}=0$, and $C_{\lambda}=L_{\lambda}^{\min }$ assumptions.

\begin{tabular}{lcccc}
\hline \hline Name & $\begin{array}{c}N \\
\left(\mathrm{year}^{-1}\right)\end{array}$ & $\begin{array}{c}E \\
\left(\times 10^{50} \mathrm{erg}\right)\end{array}$ & $\begin{array}{c}N \\
\left(\mathrm{year}^{-1}\right)\end{array}$ & $\begin{array}{c}E \\
\left(\times 10^{50} \mathrm{erg}\right)\end{array}$ \\
& \multicolumn{2}{c}{$C_{\lambda}=0$} & \multicolumn{2}{c}{$C_{\lambda}=L_{\lambda}^{\text {min }}$} \\
\hline Mrk 335 & $160.76 \pm 123.78$ & $0.25 \pm 0.26$ & $17.82 \pm 41.22$ & $0.72 \pm 0.76$ \\
Mrk 509 & $39.28 \pm 31.89$ & $2.03 \pm 0.67$ & $9.09 \pm 15.34$ & $5.08 \pm 1.64$ \\
Mrk 926 & $24.84 \pm 17.20$ & $3.94 \pm 0.87$ & $14.66 \pm 13.22$ & $6.12 \pm 1.30$ \\
Mrk 1095 & $149.32 \pm 90.68$ & $0.26 \pm 0.08$ & $18.45 \pm 31.88$ & $0.99 \pm 0.35$ \\
NGC 3516 & $104.90 \pm 23.42$ & $0.024 \pm 0.002$ & $75.44 \pm 19.86$ & $0.037 \pm 0.004$ \\
NGC 3783 & $50.11 \pm 48.48$ & $0.08 \pm 0.02$ & $28.21 \pm 36.37$ & $0.11 \pm 0.03$ \\
NGC 4151 & $32.12 \pm 8.62$ & $0.05 \pm 0.002$ & $31.16 \pm 8.49$ & $0.056 \pm 0.002$ \\
NGC 4593 & $10.32 \pm 7.09$ & $0.08 \pm 0.02$ & $2.32 \pm 3.36$ & $0.19 \pm 0.05$ \\
NGC 5548 & $51.59 \pm 18.49$ & $0.20 \pm 0.03$ & $29.52 \pm 13.99$ & $0.30 \pm 0.05$ \\
NGC 7469 & $1133.8 \pm 543.73$ & $0.02 \pm 0.01$ & $269.88 \pm 265.39$ & $0.04 \pm 0.02$ \\
3C 120.0 & $27.53 \pm 12.42$ & $0.45 \pm 0.48$ & $13.22 \pm 8.61$ & $0.65 \pm 0.67$ \\
3C 273 & $62.44 \pm 14.51$ & $71.20 \pm 27.34$ & $10.24 \pm 5.88$ & $189.62 \pm 53.83$ \\
3C 390.3 & $8.94 \pm 4.82$ & $1.64 \pm 0.49$ & $5.86 \pm 3.90$ & $2.49 \pm 0.78$ \\
Fairall 9 & $8.82 \pm 6.44$ & $11.11 \pm 1.49$ & $6.50 \pm 5.53$ & $13.03 \pm 1.74$ \\
ESO 141-55 & $9.75 \pm 5.11$ & $9.38 \pm 1.85$ & $2.69 \pm 2.69$ & $20.03 \pm 3.86$ \\
\hline
\end{tabular}

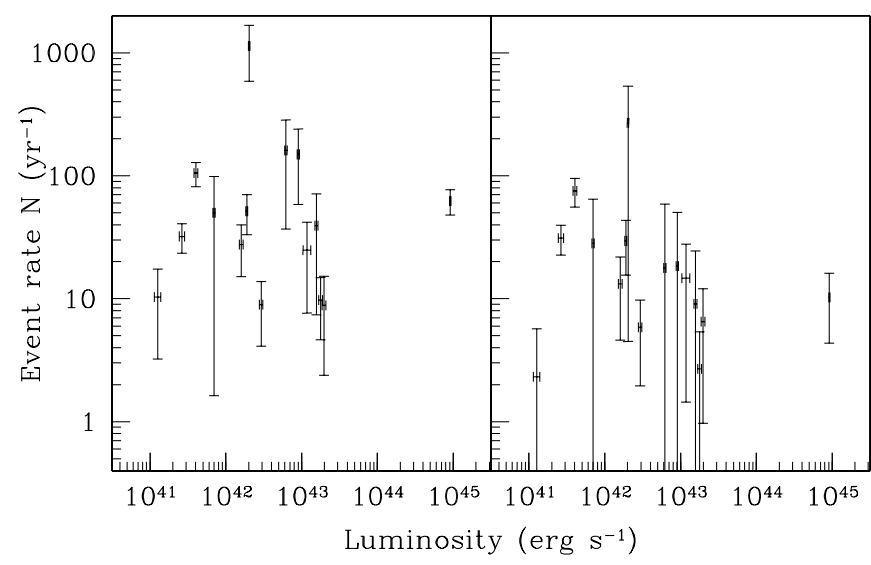

Fig. 10. Event rate as a function of the object luminosity in the hypothesis $C_{\lambda}=0$ (left panel), and using the upper limit of $C_{\lambda}$ (right panel).

correlation coefficient: $s=0.84$ ) with an index $\gamma=0.97 \pm 0.11$. These values are consistent with the non-dereddened values.

The event rates as a function of the object luminosity are given in Fig. 10, for both the upper and lower limits on $C_{\lambda}$. A non-significant anticorrelation is found in the $C_{\lambda}=0$ case (Spearman's $s=-0.12$, Null hypothesis probability of $66.64 \%$ ), while a marginally significant anticorrelation is found for $C_{\lambda}=L_{\lambda}^{\mathrm{min}}$ (Spearman's $s=-0.39$, Null hypothesis probability of $15 \%$ ). Using the data corrected for reddening, one finds as well a non-significant anticorrelation (Spearman's $s=-0.02$, Null hypothesis probability of non-correlation of $93.96 \%$ ) in the case $C_{\lambda}=0$, and a non-significant anticorrelation for $C_{\lambda}=L_{\lambda}^{\min }$ (Spearman's $s=-0.17$, Null hypothesis probability of $54.12 \%$ ).
Table 4. Slope $\eta$ of the variability-luminosity relation for the two $C_{\lambda}$ assumptions (see text).

\begin{tabular}{cccc}
\hline \hline & $\gamma$ & $\delta$ & $\eta$ \\
\hline$C_{\lambda}=0$ & $0.96 \pm 0.09$ & $0.21 \pm 0.11$ & $-0.13 \pm 0.06$ \\
$C_{\lambda}=L_{\lambda}^{\min }$ & $1.02 \pm 0.11$ & $0.21 \pm 0.11$ & $-0.10 \pm 0.05$ \\
\hline
\end{tabular}

\subsection{Constraints on the variability-luminosity relation}

Paltani \& Courvoisier (1994) showed that the variability of a similar sample was anticorrelated to the object luminosity. PC97 confirmed this result in the rest-frame of the objects and found $\sigma_{1250}^{\text {rest }} \sim \overline{L_{1250}}$, with $\eta=-0.08 \pm 0.16$.

We showed in Sect. 5.1 that the event duration $2 \mu_{1300}$ is a shallow function of the luminosity and in Sect. 5.3 that the measured event energy $E \propto{\overline{L_{1300}}}^{\gamma}$, with $\gamma \simeq 1$. These two results, expectedly, lead to values of $\eta$ in agreement with the measure of PC97. We thus established that the event parameter which drives the $\sigma(L)$ dependence is the event energy, and not its duration, nor its rate.

PC97 showed that in this case one should expect a variability-luminosity relation in the form:

$\sigma(L) \propto{\overline{L_{1300}}}^{\frac{\gamma-\delta}{2}-\frac{1}{2}}$.

We present in Table 4 the different values of the slope $\eta$ of the variability-luminosity relationship which are consistent with the value of PC97.

\section{Discussion}

\subsection{Event duration and black-hole physical time scales}

We have shown that a characteristic variability time scale exists, which can be measured in the light curves. It can be 


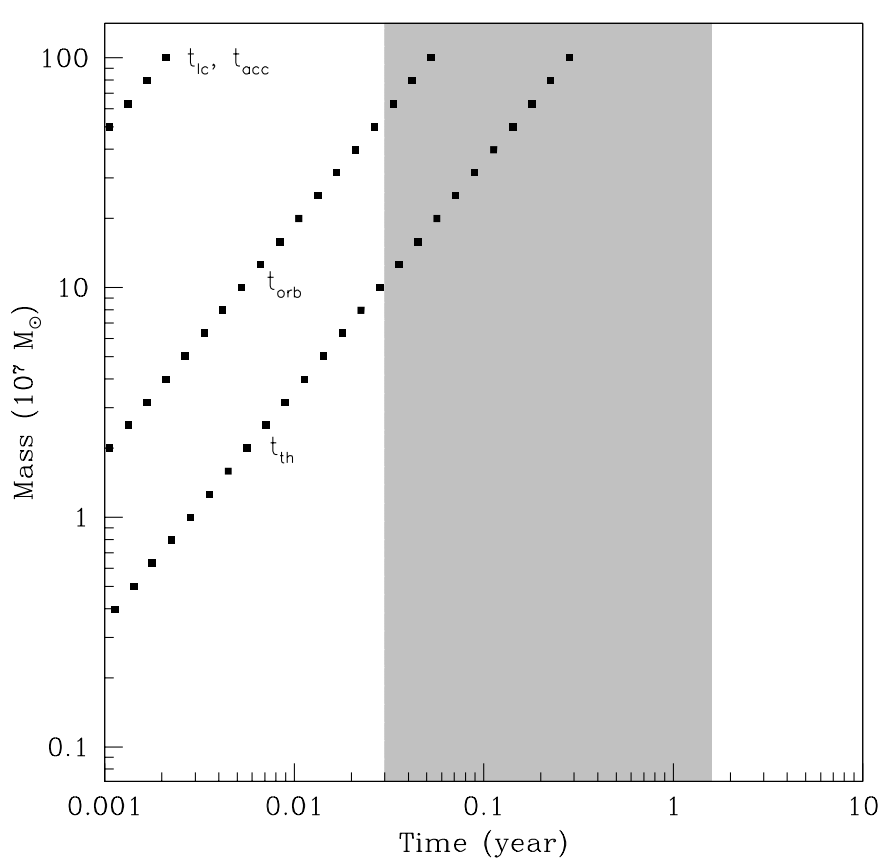

Fig. 11. Time scales vs. black hole mass. The gray area shows the range of timescales found in this study.

associated with the event duration in a model-independent way. We have obtained event durations in the range 0.03 to 1.6 years, which may possibly be related to the four physical time scales associated to black holes. They all depend on the black hole mass $M_{\mathrm{BH}}$ and Schwarzschild radius $R_{\mathrm{S}}$. We review them below, from the fastest to the slowest, following Edelson \& Nandra (1999) and Manmoto et al. (1996).

1. The light crossing time is given by $t_{\mathrm{lc}}=3.01 \times$ $10^{-5} M_{7}\left(R / 10 R_{\mathrm{S}}\right)$ year;

2. the ADAF accretion time scale (comparable to free-fall velocity) is given by $t_{\mathrm{acc}} \geq 4.38 \times 10^{-3} M_{7}\left(R / 100 R_{\mathrm{S}}\right)^{3 / 2}$ year;

3. the gas orbital time scale is given by $t_{\text {orb }}=9.03 \times$ $10^{-4} M_{7}\left(R / 10 R_{\mathrm{S}}\right)^{3 / 2}$ year;

4. the accretion disk thermal time scale $t_{\text {th }}=1.45 \times$ $10^{-2}\left(0.01 / \alpha_{\text {visc }}\right) M_{7}\left(R / 10 R_{\mathrm{S}}\right)^{3 / 2}$ year,

where $R$ is the emission distance from the center of mass, $M_{7}=M / 10^{7} M_{\odot}$, and the Schwarzschild radius is defined $R_{\mathrm{S}}=2 G M / c^{2} \cdot t_{\text {th }}$ and $t_{\text {orb }}$ can produce the range of time scales observed here for reasonable black hole masses (see Fig. 11). However, all these time scales depend linearly on the mass of the object, hence on the luminosity. The lack of strong dependence of the event duration on the object luminosity allows us to exclude all these mechanisms as likely candidates for the origin of the variability.

\subsection{Physical nature of the events}

\subsubsection{Supernovae}

In the starburst model (Aretxaga et al. 1997), the variability of AGN is produced by supernovae ( $\mathrm{SNe}$ ) explosions and compact supernovae remnants (CSNRs). The SNe generate the CSNR in the interaction of their ejecta with the stellar wind from the progenitor. Terlevich et al. (1992) showed that the properties of CSNR match the properties of the broad-line region of AGN.

Aretxaga \& Terlevich $(1993,1994)$ modeled the B band variability of the Seyfert galaxies NGC 4151 and NGC 5548 with this model. For NGC 4151, an event rate of $0.2-$ 0.3 events year $^{-1}$ was found. However, typical predictions of the model are more of the order of 3-200 events year ${ }^{-1}$ (Aretxaga et al. 1997), consistent with what we found. The event energy has to be constant $\left(3-5 \times 10^{51} \mathrm{erg}\right.$; e.g. Aretxaga et al. 1997), in clear contradiction with our result. The lifetime of CSNR (0.2-3.8 years) is compatible with the event durations found here. But no correlation with the object luminosity is expected, the more luminous objects simply having higher SNe rates. Again, this is contrary to our results. Finally, Aretxaga et al. (1997) show that a $-1 / 2$ slope of the $\sigma(L)$ relation should always be found with this model, which again is not observed.

\subsubsection{Magnetic blobs above an accretion disk}

In this model, each event is associated with the discharge of an active magnetic blob above an accretion disk. In the model proposed by Haardt et al. (1994), a fraction of the local accretion power goes into magnetic field structures allowing the formation of active blobs above the disk. Reconnection of the magnetic field lines in the corona permits the transfer of the energy into kinetic energy of fast particles. The energy is stored and released in the so-called charge and discharge times $t_{\mathrm{c}}$ and $t_{\mathrm{d}}$ with $t_{\mathrm{d}} \ll t_{\mathrm{c}}$. Using the dynamo model of Galeev et al. (1979) for the blob formation, Haardt et al. (1994) show that $t_{\mathrm{d}}$ scales with the blob size $R_{\mathrm{b}}$ which itself scales with the total luminosity $L$ of the source. This trend is clearly not seen in our data (Fig. 7). Furthermore, the total number of active loops $N_{\text {tot }}$, at any time, does not depend on the luminosity nor on the mass of the object. The blob rate $N_{\text {tot }} / t_{\mathrm{d}}$ becomes therefore proportional to $L^{-1}$, also in clear contradiction with our results that show that the event rate is not correlated with the luminosity. Finally, the energy released by a single blob can be written $E=t_{\mathrm{d}} L_{\text {blob}}$, where $L_{\text {blob }}$ is given by Eq. (7) of Haardt et al. (1994). The energy $E$ released then goes with $L^{2}$, also in contradiction with the results deduced here in which $E \propto L$.

\subsubsection{Stellar collisions}

Courvoisier et al. (1996) proposed that the energy radiated in AGN originates in a number of collisions between stars that orbit the supermassive black hole at very high velocities in a volume of some $100 R_{\mathrm{S}}$. They computed the rate $\mathrm{d} n / \mathrm{d} t$ of headon stellar collisions in a spherical shell of width $\mathrm{d} r$, located at distance $r$ from the central black hole. The stars are assumed to have mass $M_{\odot}$ and radius $R$ of the Sun. This reads:

$$
\frac{\mathrm{d} n}{\mathrm{~d} t}=\left(\rho^{2} 4 \pi r^{2} \mathrm{~d} r\right) v_{\mathrm{K}} \pi R^{2},
$$

where $\rho$ represents the stellar density and $v_{\mathrm{K}}$ the Keplerian velocity (Courvoisier et al. 1996; Torricelli-Ciamponi et al. 2000). Assuming that the stars are located in a shell of inner radius $a$ and outer radius $b$ and are distributed following a density 
law $\rho=N_{0}\left(r / r_{0}\right)^{-\alpha / 2}$, with slope $\alpha$ where $N_{0}$ and $r_{0}$ are constants, we find for the kinetic energy released by one event:

$E=\frac{\int \frac{1}{2} M_{\odot} v_{\mathrm{K}}^{2} \frac{\mathrm{d} n}{\mathrm{~d} t}}{\int \frac{\mathrm{d} n}{\mathrm{~d} t}}=\frac{\frac{1}{2} M_{\odot} \int_{a}^{b} \mathrm{~d} r v_{\mathrm{K}}^{3}(r) r^{2-\alpha}}{\int_{a}^{b} \mathrm{~d} r v_{\mathrm{K}}(r) r^{2-\alpha}}$,

where $\alpha, a$ and $b$ are parameters of the stellar cluster. Using $v_{\mathrm{K}}=\sqrt{G M_{\text {tot }} / r}$ leads to:

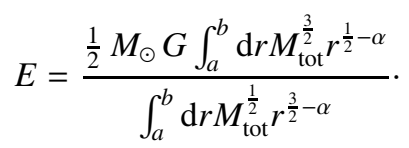

Neglecting the cluster's mass with respect to the black hole mass, i.e. making the assumption that $M_{\mathrm{tot}}(r) \sim M_{\mathrm{BH}}$, we finally have:

$E=\frac{1}{2} M_{\odot} G M_{\mathrm{BH}} f(\alpha, a, b)$,

where $f$ is a function of the cluster parameters only, independent of $M_{\mathrm{BH}}$.

We need now to relate the average luminosity to the black hole mass. This relation comes from $\bar{L}=\int 1 / 2 M_{\odot} v_{\mathrm{K}}^{2} \mathrm{~d} n / \mathrm{d} t=$ $\left(M_{\odot} R_{\odot}^{2} \pi^{2} G^{3 / 2} N_{0} r_{0}^{\alpha}\right) M_{\mathrm{BH}}^{3 / 2} f(\alpha, a, b)$. As $E \propto M_{\mathrm{BH}}$ and $\bar{L} \propto$ $M_{\mathrm{BH}}^{3 / 2}$, we finally have:

$E \propto \bar{L}^{\frac{2}{3}}$.

This relation is a relatively good approximation of the trends seen in Fig. 9, although not completely satisfactory.

In this model, the variability time scale $2 \mu_{\lambda}$ is expected to be related to the time needed to the expanding sphere to become optically thin. This point is discussed in Courvoisier \& Türler (2004) who found that for clumps of about one Solar mass, the expansion time is about $2 \times 10^{6} \mathrm{~s}$. This enters in the range of time scales found here. The collision rate should be going with $M_{\mathrm{BH}}^{1 / 2}$, which implies $N \propto \bar{L}^{1 / 3}$, which is not seen in our data.

\subsubsection{Other models}

For the sake of completeness, we note that gravitational microlensing models, in which populations of planetary mass compact bodies randomly cross the line of sight of an observed AGN, can be invoked to explain the long term variations over several years. But in the case of low-redshift Seyfert galaxies, which forms the majority of our sample, the probabilities of micro-lensing are not significant (Hawkins 2001).

Finally, a model of accretion disk instabilities has been suggested (Kawaguchi et al. 1998) to explain the optical variability of AGN. The SOC state model (Mineshige et al. 1994) is producing power density spectra in good agreement with the observations but, since it is not an event-based model, it is difficult to use the measurements discussed here to constrain it.

\section{Conclusion}

We showed the existence of a maximum variability time scale in the ultraviolet light curves of 15 type 1 AGN, in the range
$1300-3000 \AA$. We found variability time scales in the range $0.02-1.00$ year.

In the framework of the discrete-event model, we showed that these time scales can be related to the event duration in a simple manner. A weak dependence of the event duration with the object luminosity at $1300 \AA$ is found. The event duration is not a function of the wavelength in the range 1300 to $3000 \AA$.

The event energy per object varies from $10^{48}$ to $10^{52} \mathrm{erg}$ with a corresponding event rate comprised between 2 and 270 events per year, assuming the presence of a constant component in the light curves.

Our results do not depend on the constant component $C_{\lambda}$. While we can only provide lower and upper bounds on $C_{\lambda}$, its choice does not change the conclusions.

The event energy is strongly correlated with the object luminosity. We show that the combined relations of the event energy $E \propto \bar{L}_{1300}^{1.02}$, and event duration $2 \mu_{1300} \propto \bar{L}_{1300}^{0.21}$ with the object luminosity, lead to the trend seen in the variabilityluminosity relationship in the rest frame, i.e. that both variables are correlated with a slope of about 0.08 . We thus established that the event parameter which drives the $\sigma(L)$ dependence is the event energy, and not its duration, nor its rate.

These results allow us to constrain the physical nature of the events. We show that neither the starburst model nor the magnetic blob model can satisfy these requirements. On the other hand, stellar collision models in which the average properties of the collisions depend on the mass of the central black hole may be favored, although the model will need to be improved as the result we found (for instance, the lack of correlation between the event rate and the luminosity) does not match the predictions.

Acknowledgements. S.P. acknowledges a grant from the Swiss National Science Foundation.

\section{References}

Akritas, M. G., \& Bershady, M. A. 1996, ApJ, 470, 706

Aretxaga, I., \& Terlevich, R. J. 1993, Ap\&SS, 206, 69

Aretxaga, I., \& Terlevich, R. 1994, MNRAS, 269, 462

Aretxaga, I., Cid Fernandes, R., \& Terlevich, R. J. 1997, MNRAS, 286, 271

Cid Fernandes, R., Sodré, L., \& Vieira da Silva, L. 2000, ApJ, 544, 123 (CSV00)

Cid Fernandes, R. J., Aretxaga, I., \& Terlevich, R. 1996, MNRAS, 282, 1191

Collier, S., \& Peterson, B. M. 2001, ApJ, 555, 775 (CP01)

Courvoisier, T. J.-L., \& Clavel, J. 1991, A\&A, 248, 389

Courvoisier, T. J.-L., Paltani, S., \& Walter, R. 1996, A\&A, 308, L17

Courvoisier, T. J.-L., \& Türler, M. 2004, submitted

Cristiani, S., Trentini, S., La Franca, F., et al. 1996, A\&A, 306, 395

di Clemente, A., Giallongo, E., Natali, G., Trevese, D., \& Vagnetti, F. 1996, ApJ, 463, 466

Edelson, R., \& Nandra, K. 1999, ApJ, 514, 682

Galeev, A. A., Rosner, R., \& Vaiana, G. S. 1979, ApJ, 229, 318

Giveon, U., Maoz, D., Kaspi, S., Netzer, H., \& Smith, P. S. 1999, MNRAS, 306, 637

Haardt, F., Maraschi, L., \& Ghisellini, G. 1994, ApJ, 432, L95

Hawkins, M. R. S. 2001, ApJ, 553, L97 
Hook, I. M., McMahon, R. G., Boyle, B. J., \& Irwin, M. J. 1994, MNRAS, 268, 305

Kawaguchi, T., Mineshige, S., Umemura, M., \& Turner, E. L. 1998, ApJ, 504, 671

Kinney, A. L., Bohlin, R. C., Blades, J. C., \& York, D. G. 1991, ApJS, 75,645

Koratkar, A., \& Blaes, O. 1999, PASP, 111, 1

Krolik, J. H. 1999, Active galactic nuclei: from the central black hole to the galactic environment (Princeton University Press)

Manmoto, T., Takeuchi, M., Mineshige, S., Matsumoto, R., \& Negoro, H. 1996, ApJ, 464, L135

Mineshige, S., Takeuchi, M., \& Nishimori, H. 1994, ApJ, 435, L125

Paltani, S. 1996, Ph. D. Thesis, Geneva University

Paltani, S. 1999, in BL Lac Phenomenon, ASP Conf. Ser., 159, 293

Paltani, S., \& Courvoisier, T. J.-L. 1994, A\&A, 291, 74

Paltani, S., \& Courvoisier, T. J.-L. 1997, A\&A, 323, 717 - PC97
Paltani, S., \& Walter, R. 1996, A\&A, 312, 55

Paltani, S., Courvoisier, T. J.-L., \& Walter, R. 1998, A\&A, 340, 47

Rodríguez-Pascual, P. M., González-Riestra, R., Schartel, N., \& Wamsteker, W. 1999, A\&AS, 139, 183

Simonetti, J. H., Cordes, J. M., \& Heeschen, D. S. 1985, ApJ, 296, 46 Türler, M., Paltani, S., Courvoisier, T. J.-L., et al. 1999, A\&AS, 134, 89

Terlevich, R., Tenorio-Tagle, G., Franco, J., \& Melnick, J. 1992, MNRAS, 255, 713

Torricelli-Ciamponi, G., Foellmi, C., Courvoisier, T. J.-L., \& Paltani, S. 2000, A\&A, 358, 57

Trèvese, D., \& Vagnetti, F. 2002, ApJ, 564, 624

Trevese, D., Kron, R. G., Majewski, S. R., Bershady, M. A., \& Koo, D. C. 1994, ApJ, 433, 494

Welsh, W. F. 1999, PASP, 111, 1347 
P. Favre et al.: AGN variability time scales and the discrete-event model, Online Material p 1

\section{Online Material}


P. Favre et al.: AGN variability time scales and the discrete-event model, Online Material p 2

Appendix A: Structure functions 1300-3000 
P. Favre et al.: AGN variability time scales and the discrete-event model, Online Material p 3
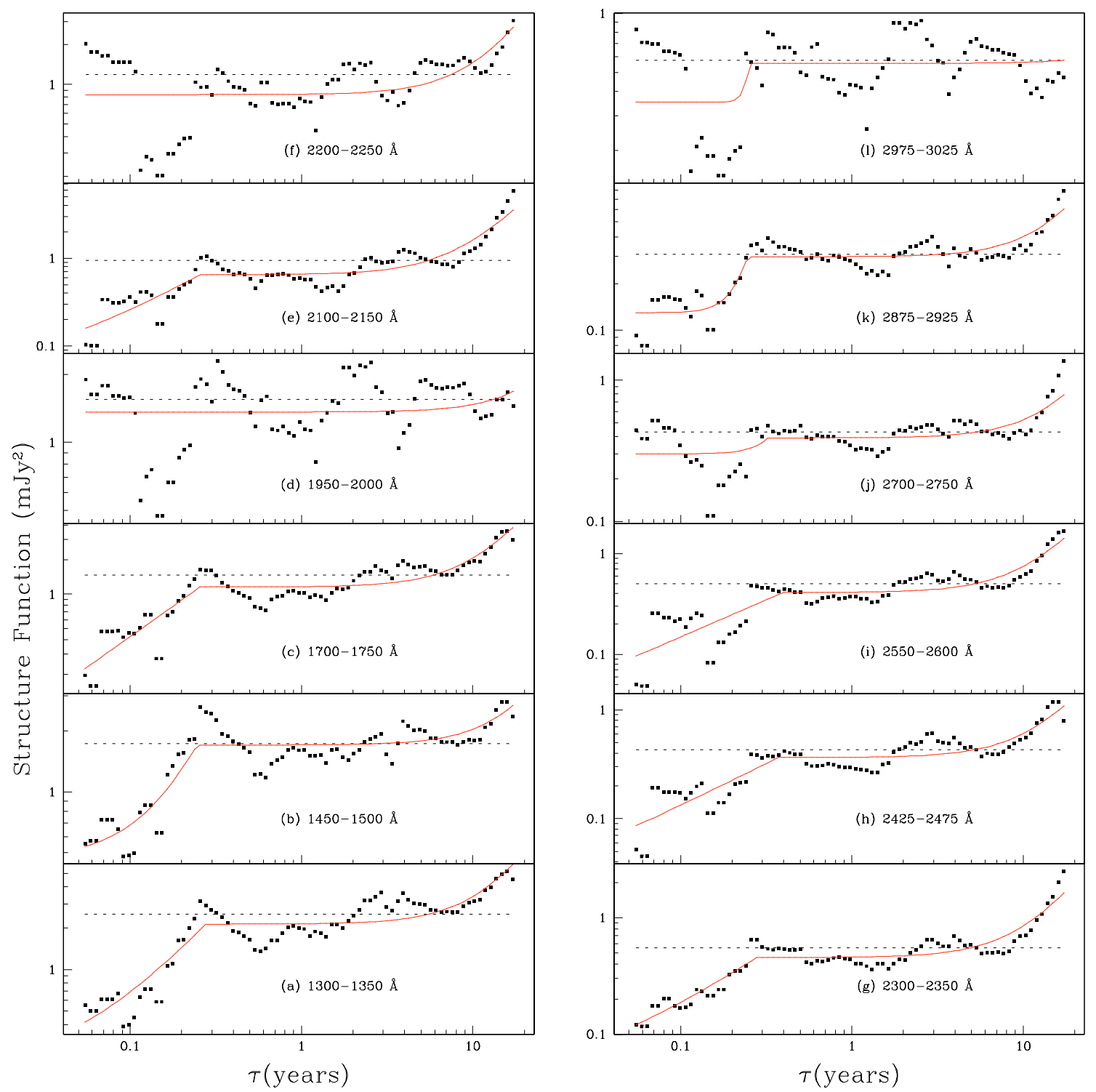

Fig. A.1. Mrk 335, SF for the light curves 1300 to $3000 \AA$. The fit did not succeed for the SF (d), (f), (j), (1), because of noisy light curves (see Figs. A.2-A.5). The time scales corresponding to the SF (d), (f), (j), (1) are thus rejected. 
P. Favre et al.: AGN variability time scales and the discrete-event model, Online Material p 4

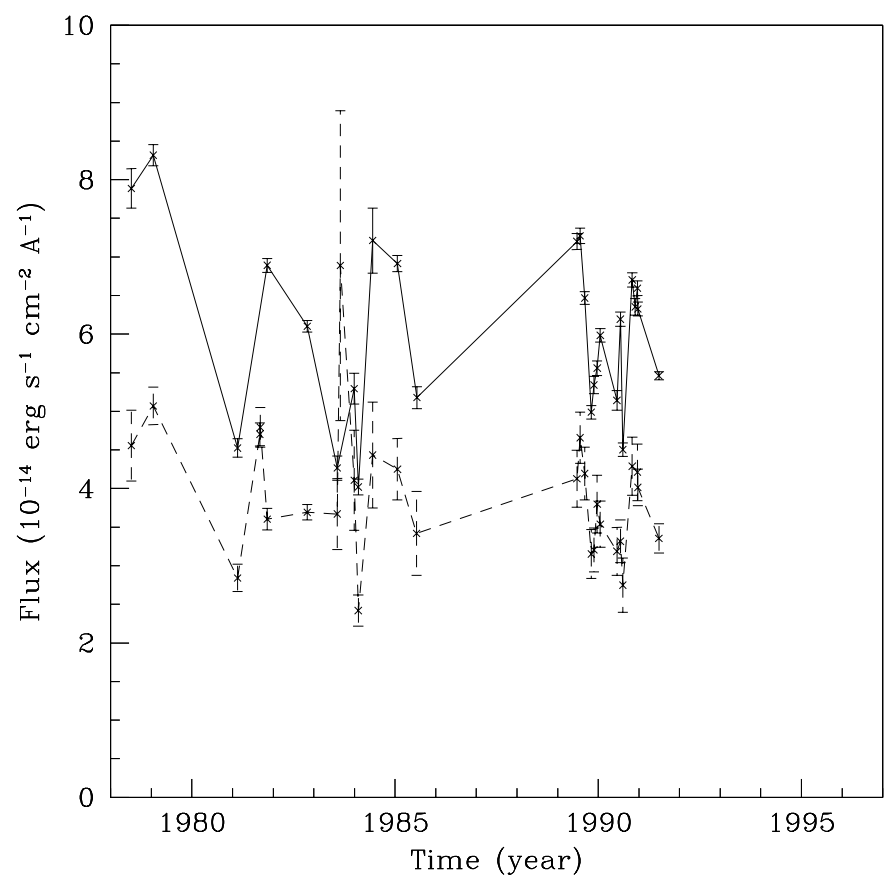

Fig. A.2. Mrk 335, comparison of the noisy light curve 1950-2000 (dashed line) with the 1300-1350 ̊ light curve.

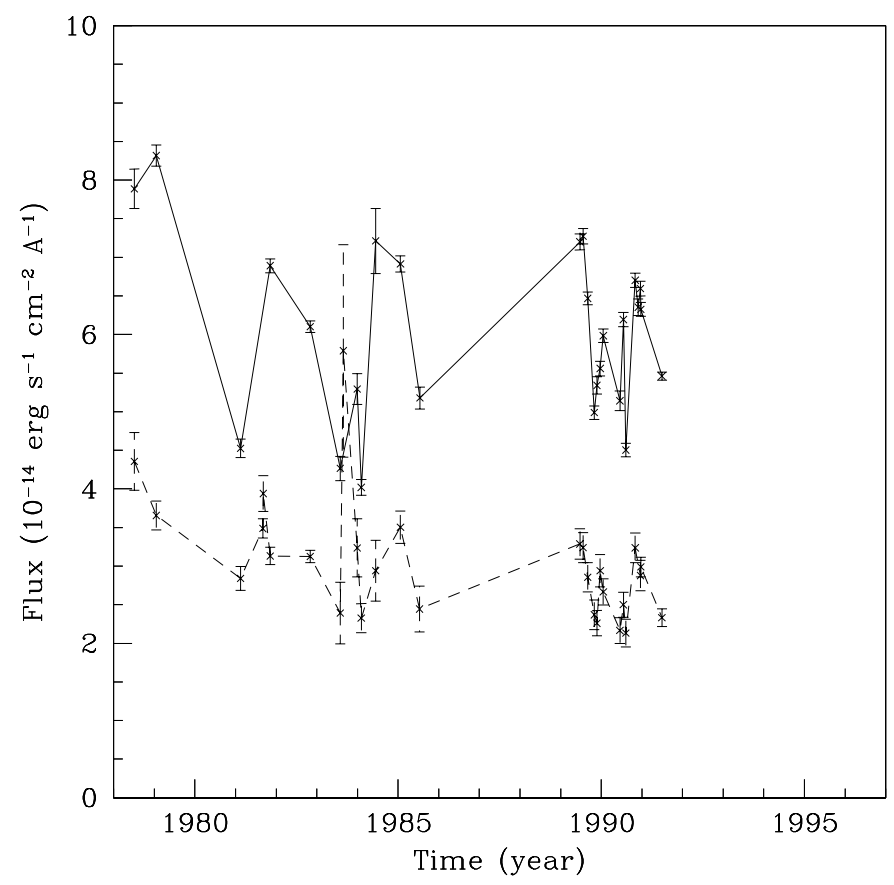

Fig. A.3. Mrk 335, comparison of the noisy light curve 2200-2250 ̊ (dashed line) with the 1300-1350 ̊ light curve.

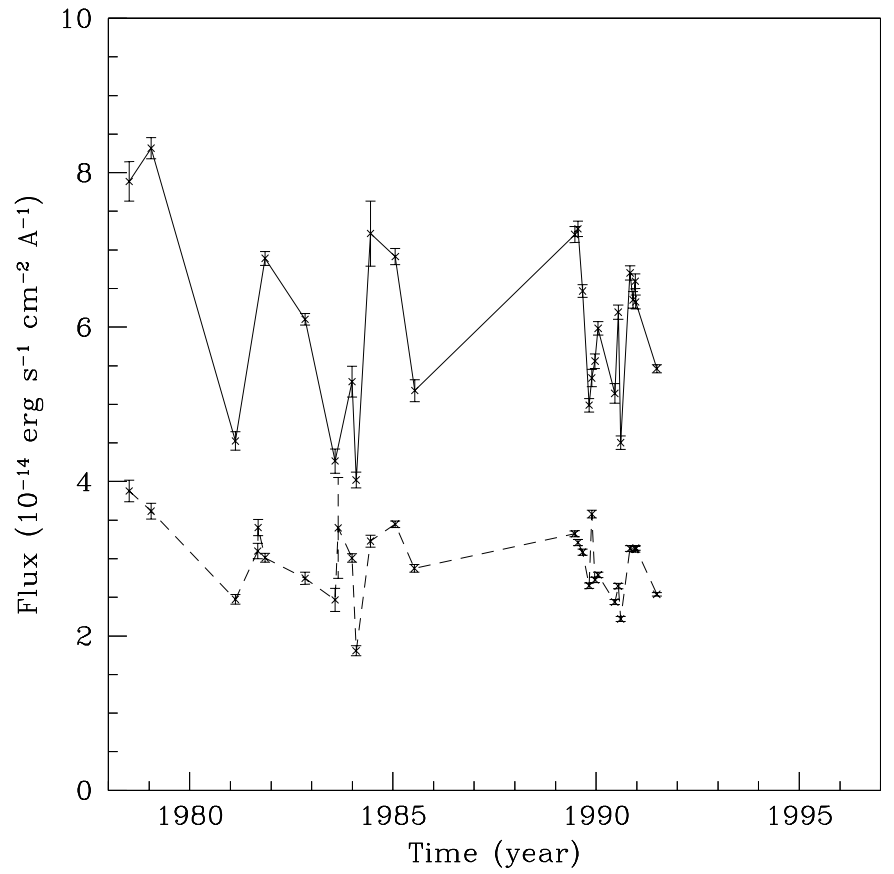

Fig. A.4. Mrk 335, comparison of the noisy light curve 2700-2750 ̊ (dashed line) with the 1300-1350 A light curve.

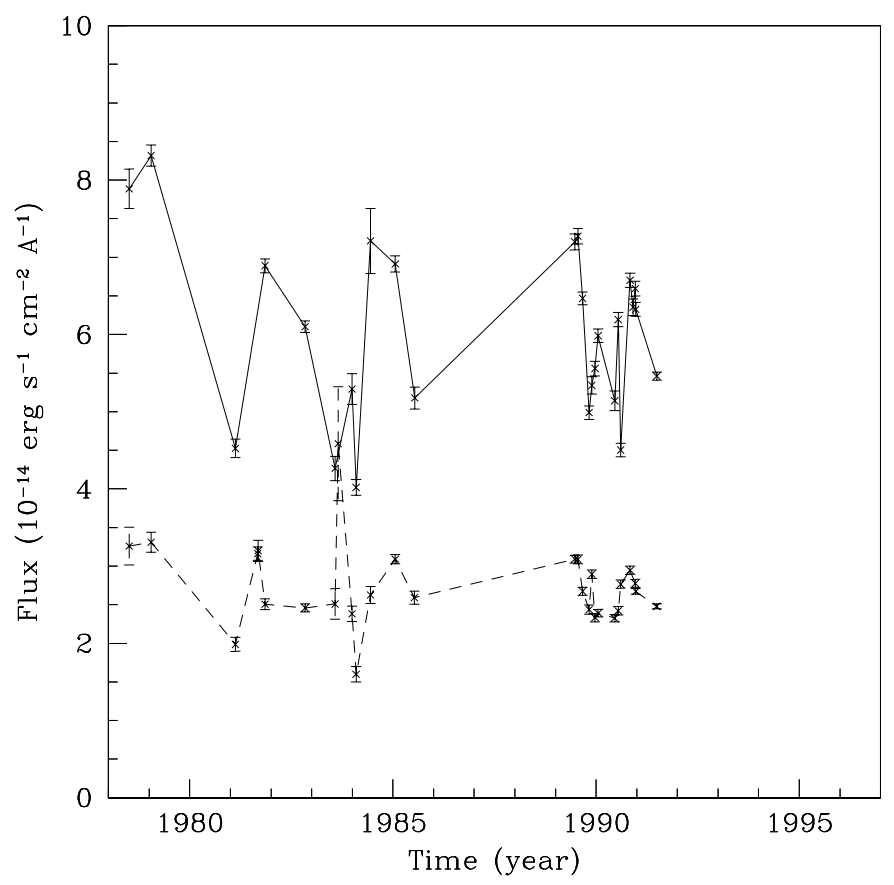

Fig. A.5. Mrk 335, comparison of the noisy light curve 2975-3025 (dashed line) with the 1300-1350 A light curve. 
P. Favre et al.: AGN variability time scales and the discrete-event model, Online Material p 5
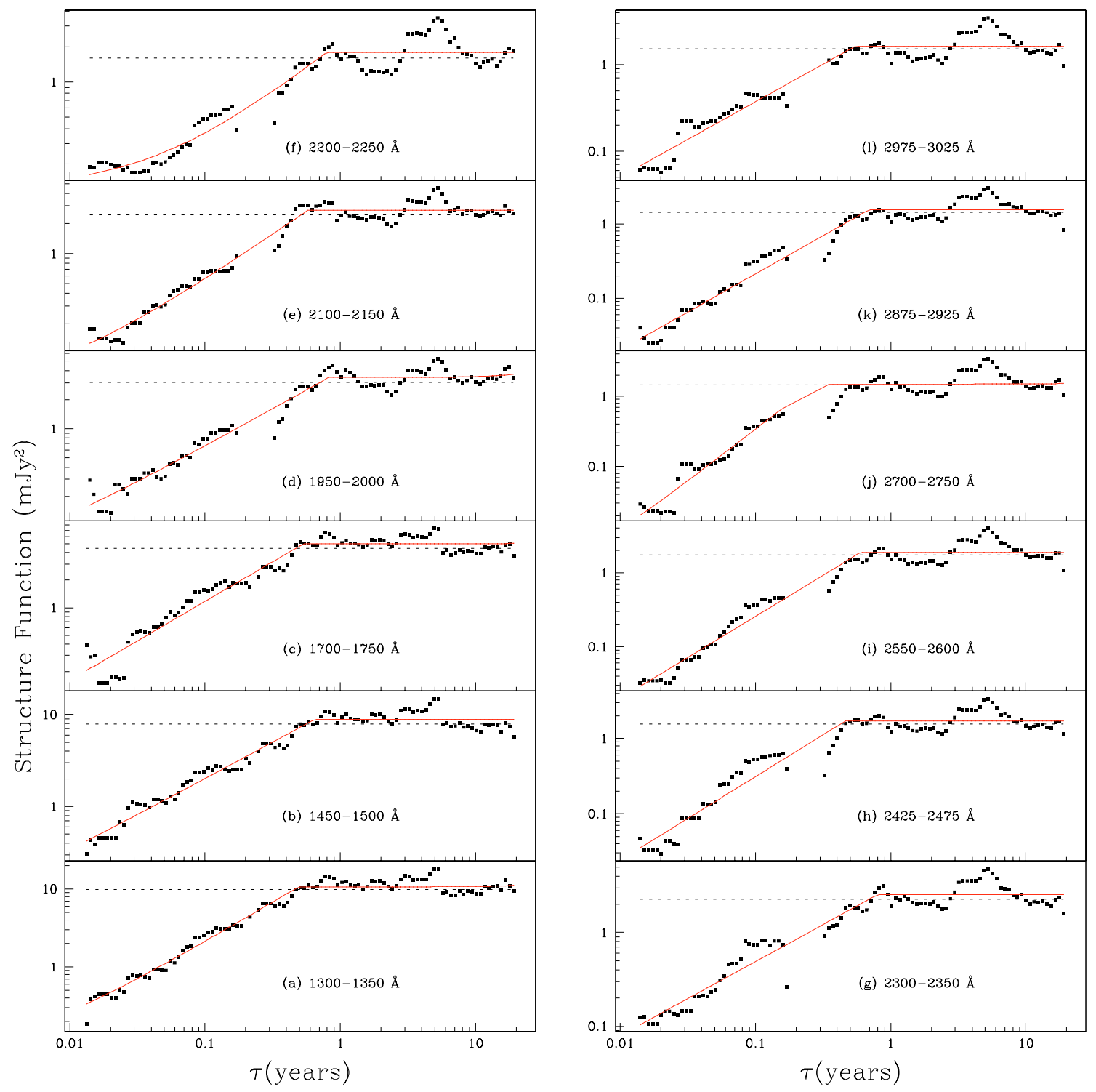

Fig. A.6. Mrk 509, SF for the light curves 1300 to $3000 \AA$. 
P. Favre et al.: AGN variability time scales and the discrete-event model, Online Material $p 6$
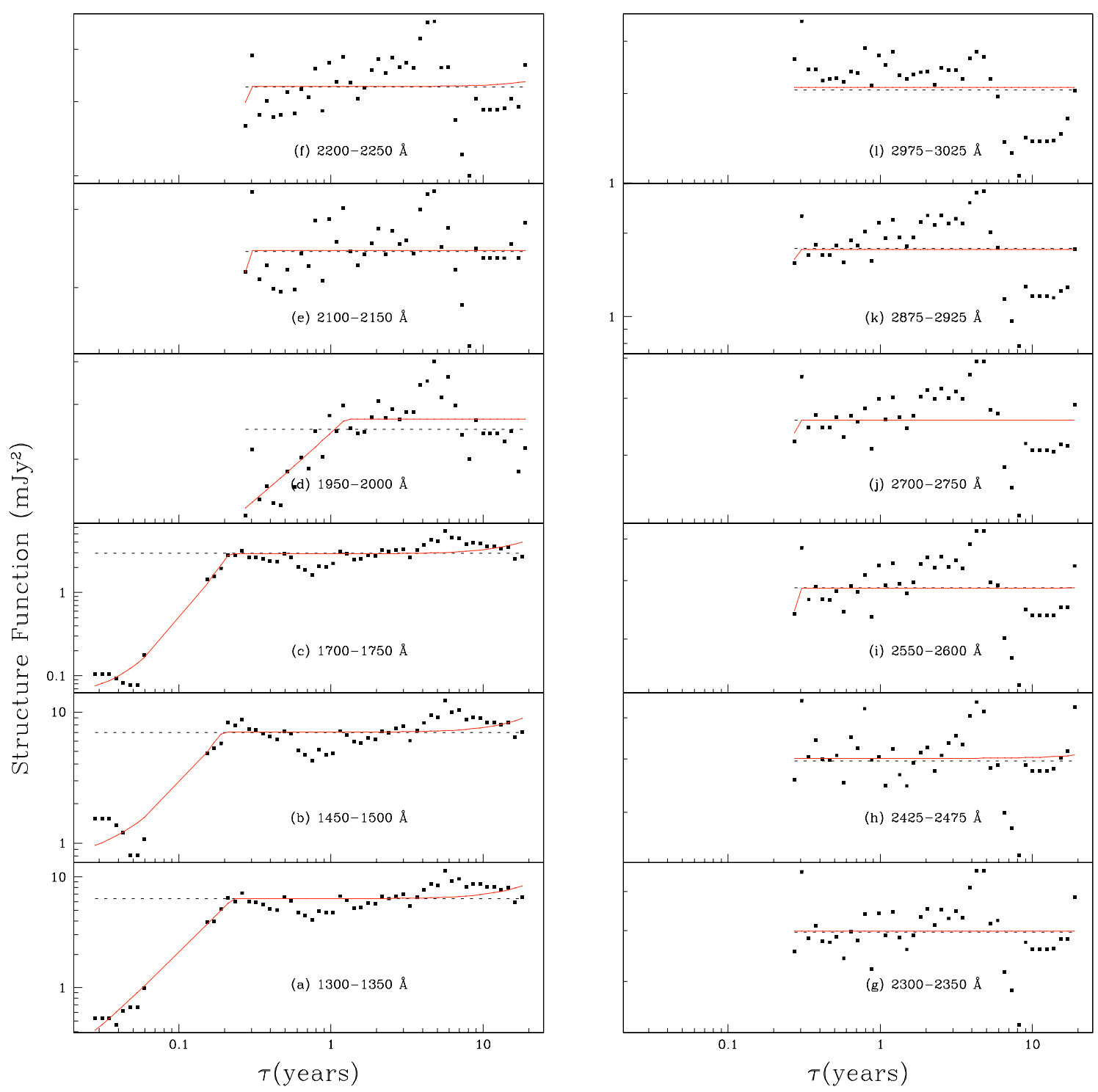

Fig. A.7. Mrk 926, SF for the light curves 1300 to $3000 \AA$ A. The fit did not succeed for the all the SF built from data taken with the LW instrument (1950-3025 $\AA$ ). The reason is the small number of epochs (16) taken with the LW (see Fig. A.8). We thus reject the time scales corresponding to the $\mathrm{SF}(\mathrm{d})-(\mathrm{l})$. 
P. Favre et al.: AGN variability time scales and the discrete-event model, Online Material p 7

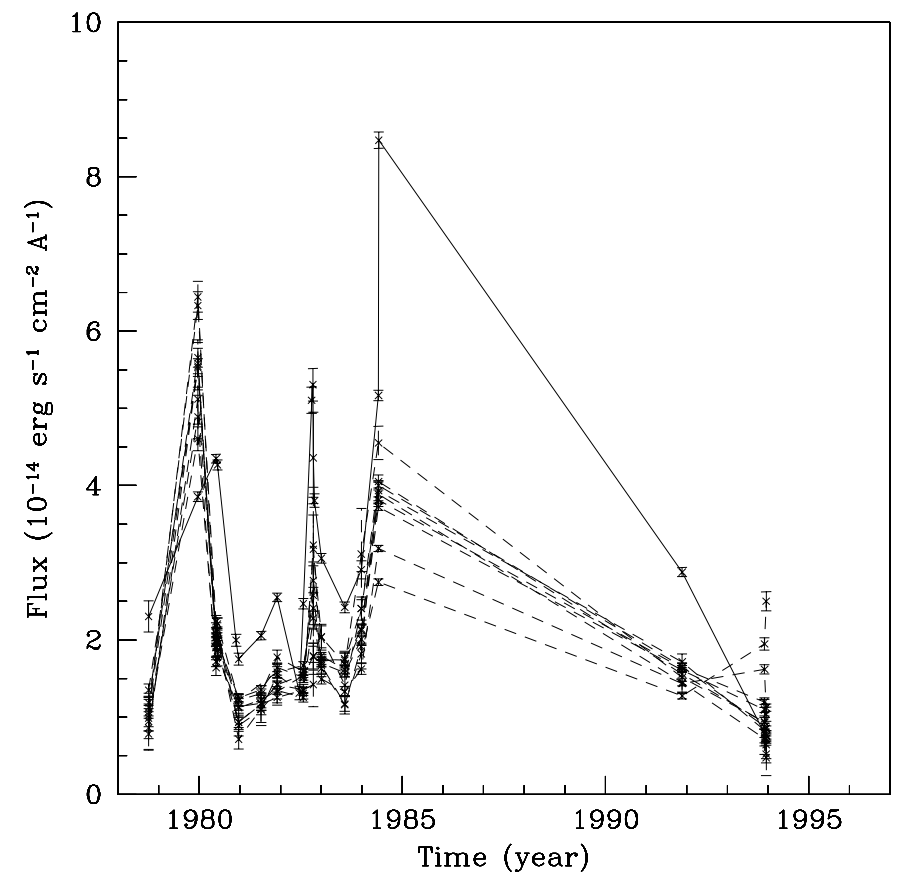

Fig. A.8. Mrk 926, comparison of the LW light curves (dashed lines) with the 1300-1350 ̊̊ light curve. 
P. Favre et al.: AGN variability time scales and the discrete-event model, Online Material $p 8$
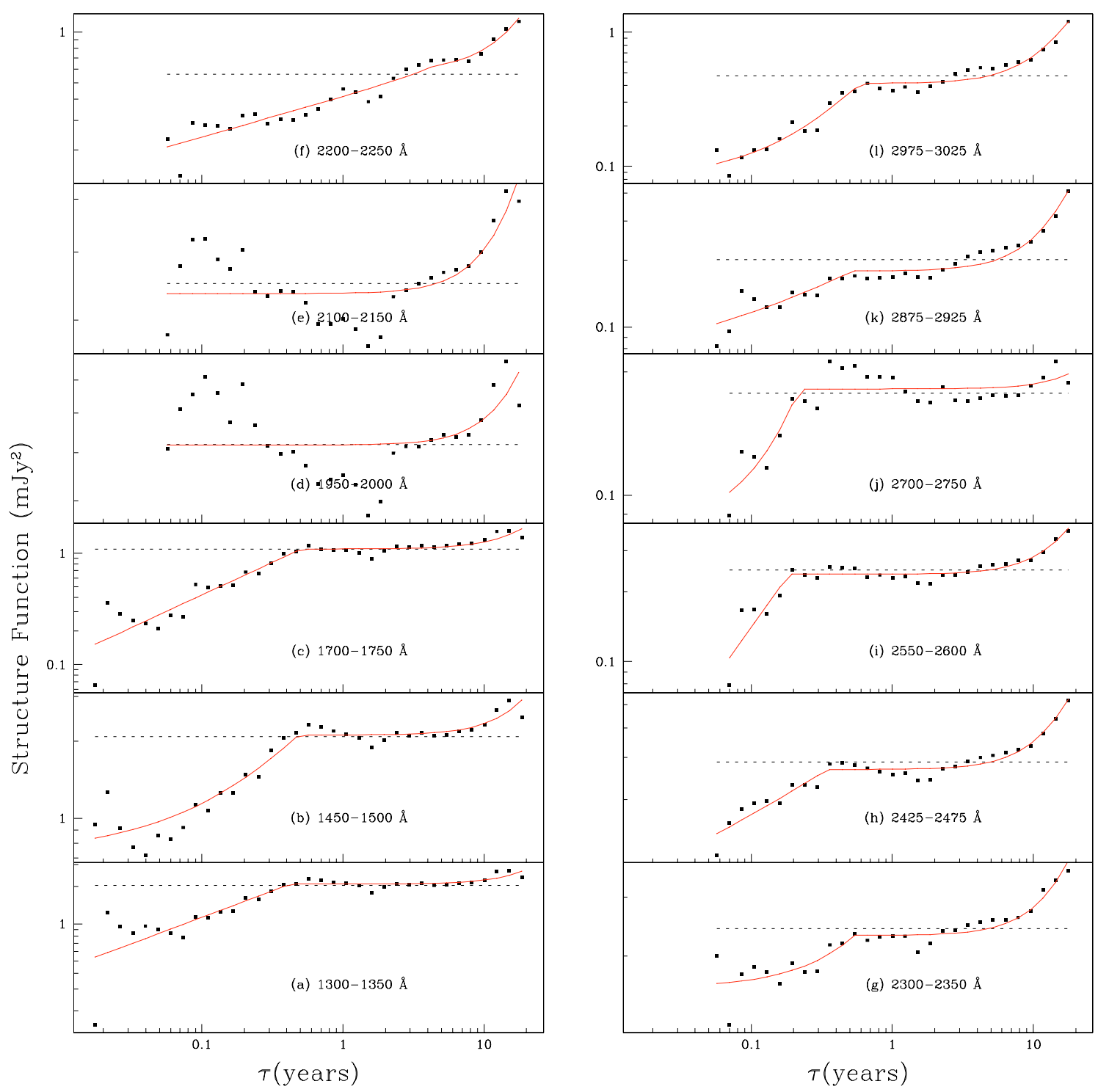

Fig. A.9. Mrk 1095, SF for the light curves 1300 to $3000 \AA$ A. The fit did not succeed for the SF (d), (e), (f), because of noisy light curves (see Fig. A.10). The time scales corresponding to the SF (d), (e), (f) are thus rejected. 
P. Favre et al.: AGN variability time scales and the discrete-event model, Online Material $p 9$

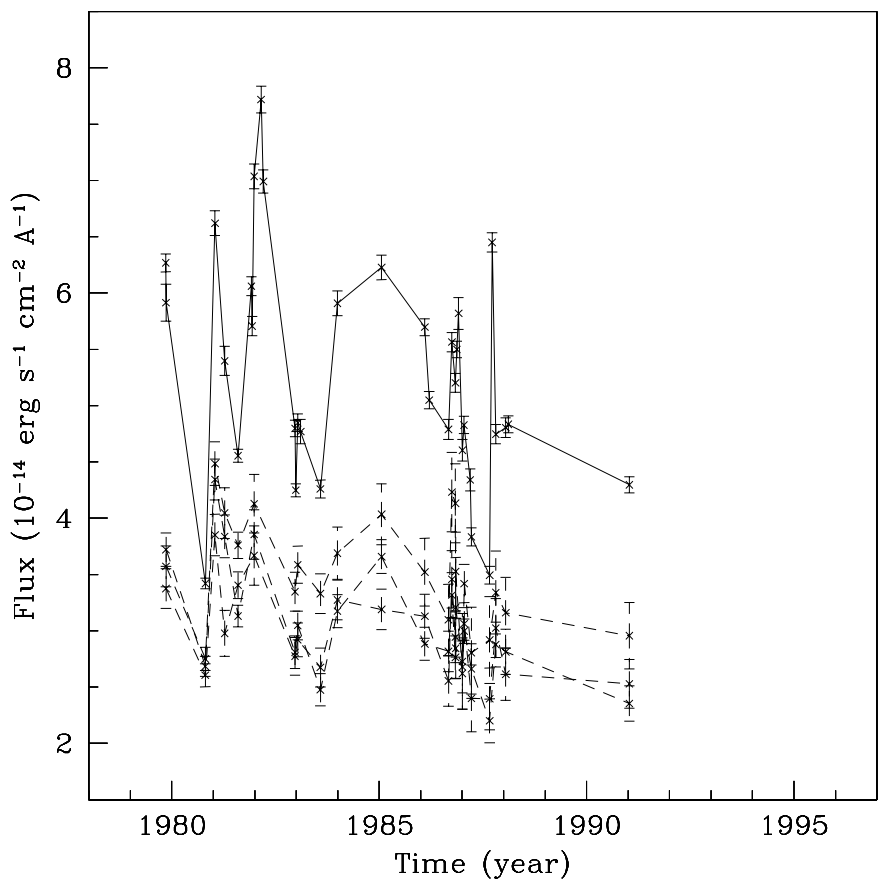

Fig. A.10. Mrk 1095, comparison of the noisy 1950-2000, 21002150, 2200-2250 light curves (dashed lines) with the 1300-1350 ̊ light curve. 
P. Favre et al.: AGN variability time scales and the discrete-event model, Online Material $p 10$
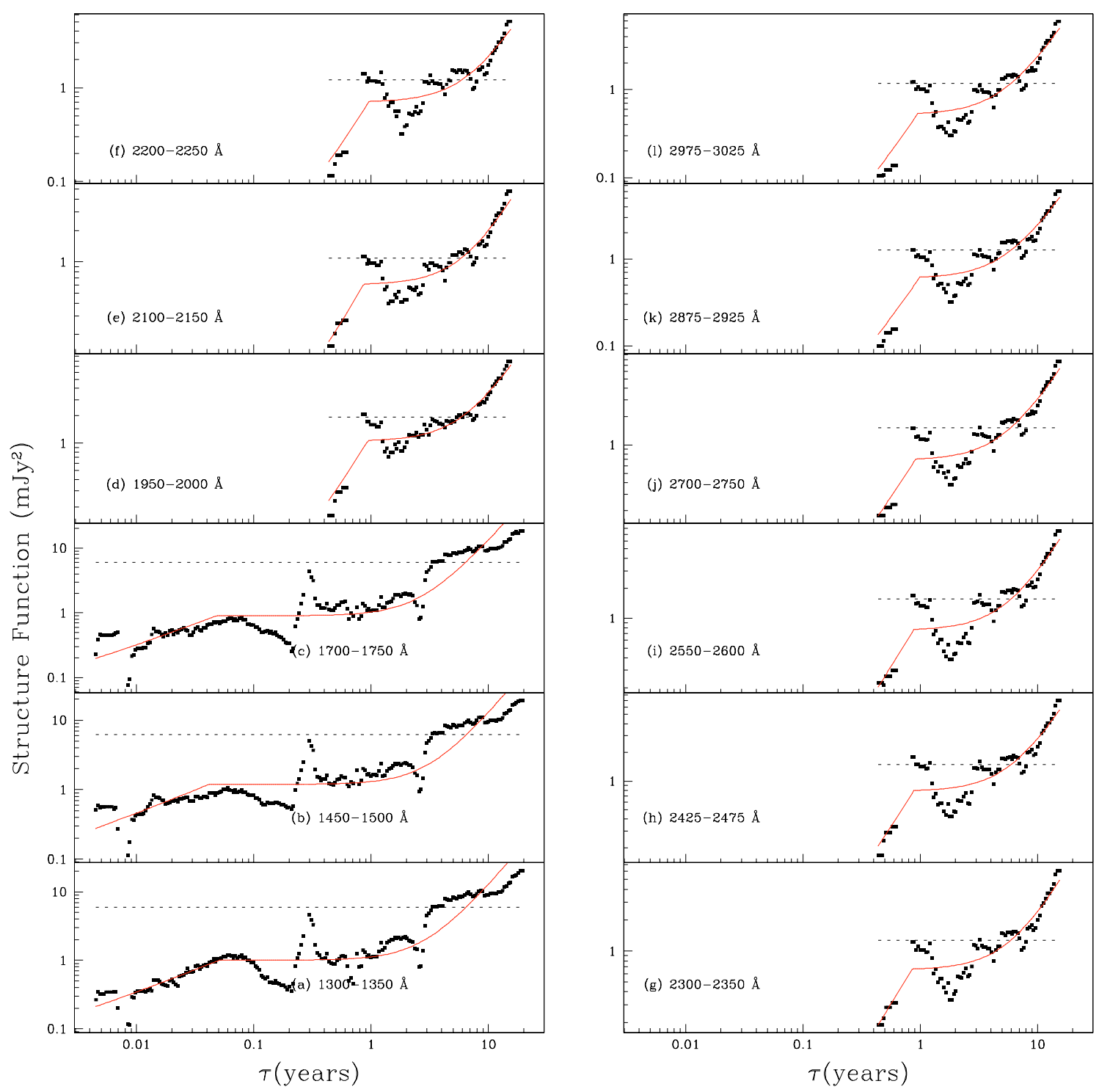

Fig. A.11. NGC 3516, SF for the light curves 1300 to $3000 \AA$ A. The fit of the SF (a)-(c) follows what was done in Sect. 4.1 (two parameters fixed). The LW was less used than the SWP during the intensive monitoring made in 1993 (see Fig. A.12). The number of LW epochs is smaller (22) than for the SWP (71). Although the fit seems to have succeeded in the LW range, one should be careful in interpreting the result as evidence for the presence of a longer timescale as the SF slope $\alpha$ pegged to the maximum value of 2 . In addition, the fit is rather poor. We thus decided to reject the time scales corresponding to the SF (d)-(l). 
P. Favre et al.: AGN variability time scales and the discrete-event model, Online Material p 11

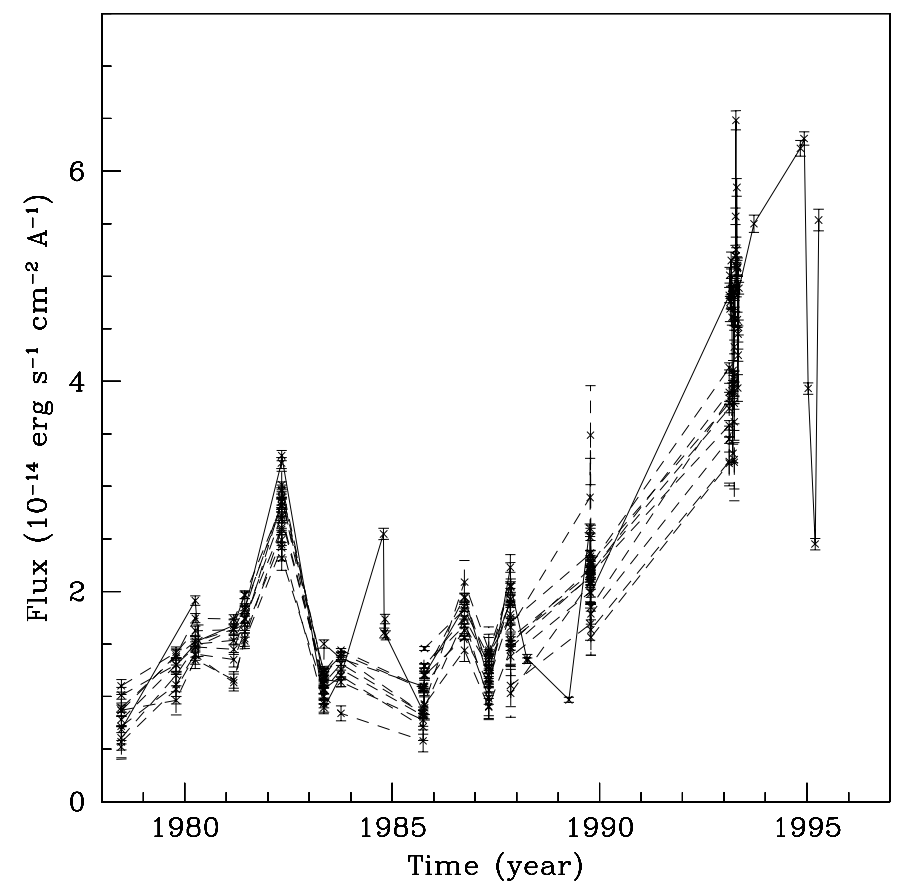

Fig. A.12. NGC 3516, comparison of the LW light curves (dashed lines) with the 1300-1350 A light curve. 
P. Favre et al.: AGN variability time scales and the discrete-event model, Online Material p 12
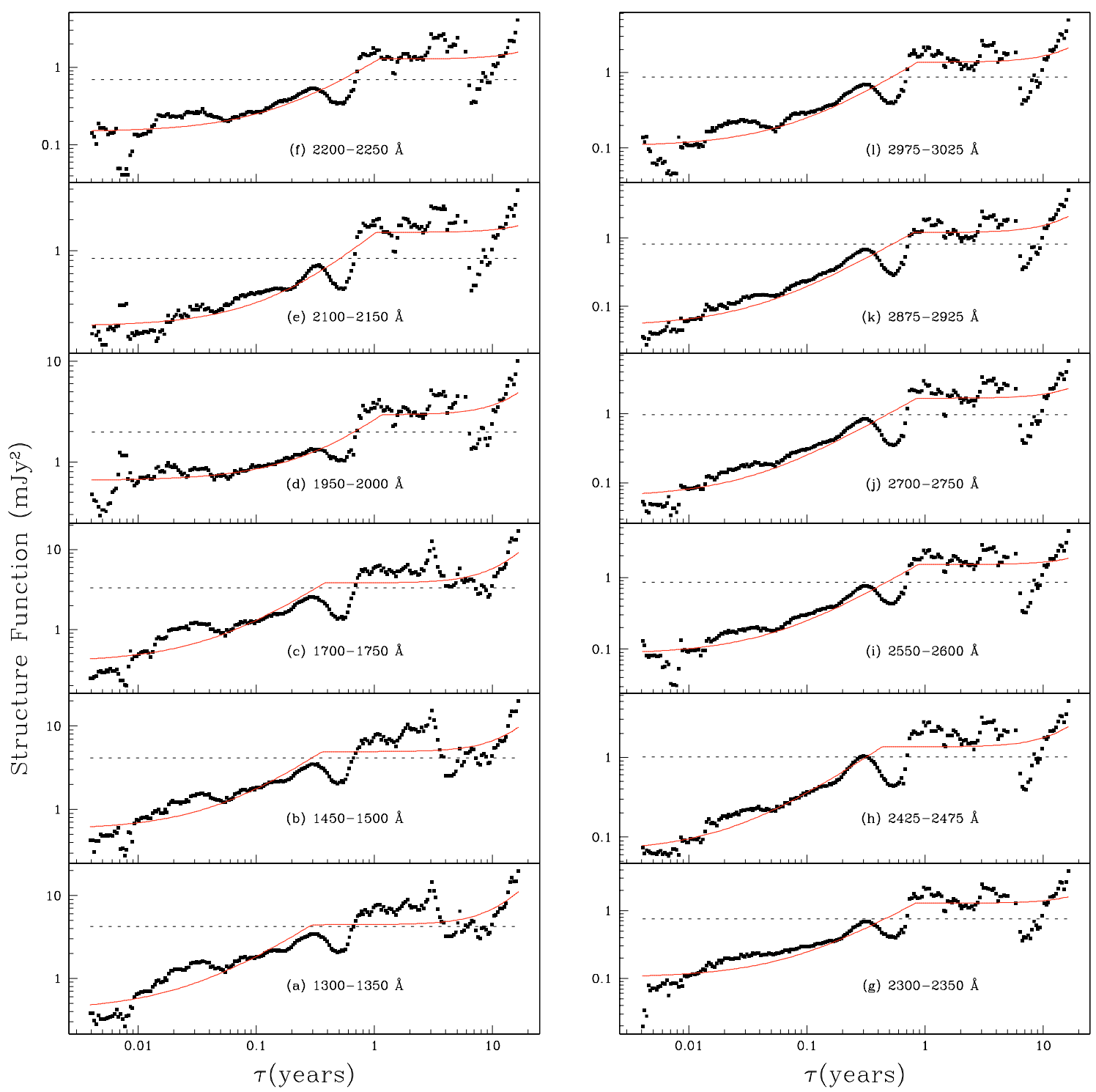

Fig. A.13. NGC 3783, SF for the light curves 1300 to $3000 \AA$ A. The time scales obtained from the LW data are more scattered because the LW data have $12 \%$ less epochs than the SWP. 
P. Favre et al.: AGN variability time scales and the discrete-event model, Online Material $p 13$
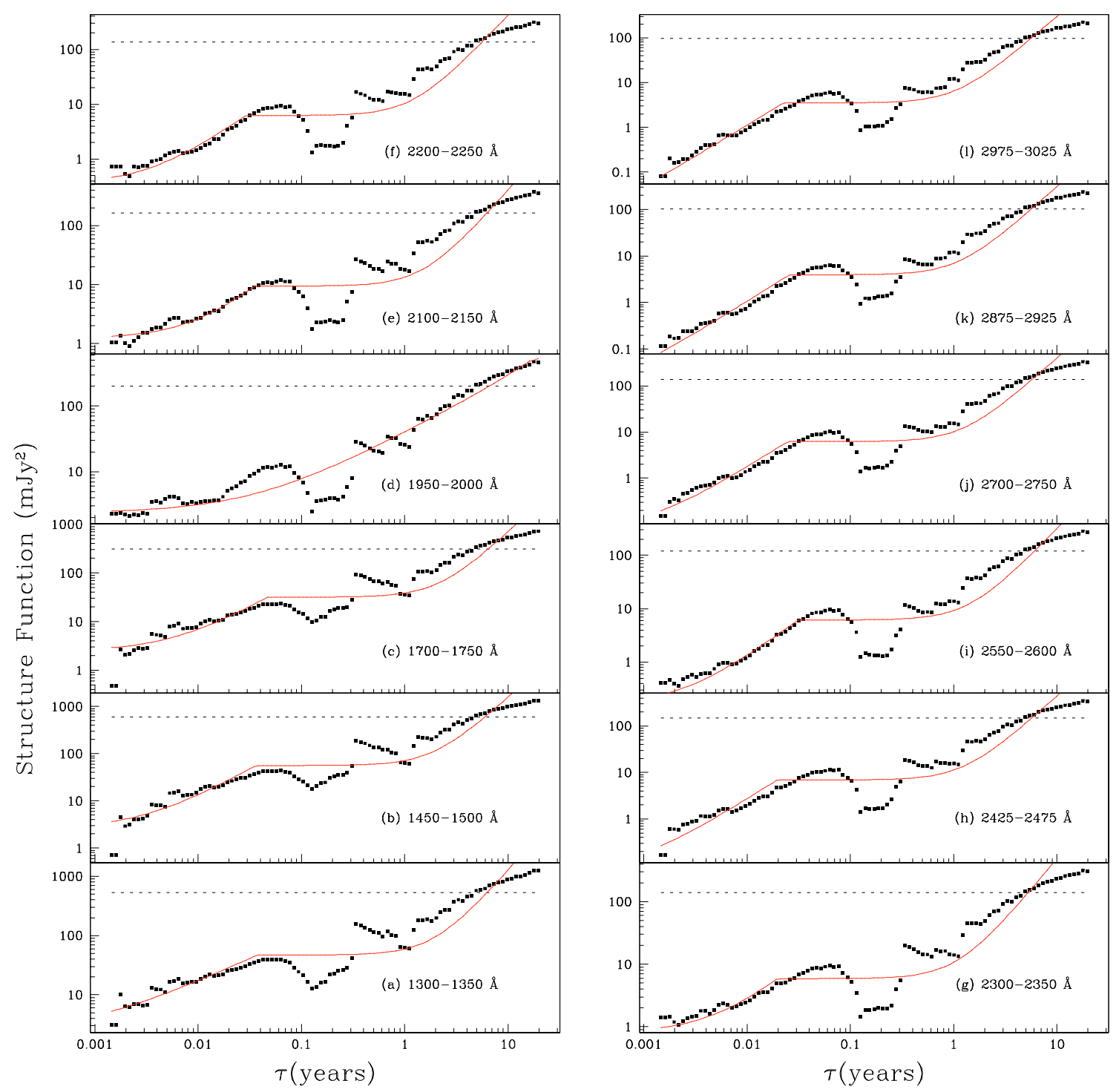

Fig. A.14. NGC 4151, SF for the light curves 1300 to $3000 \AA$ A. The fit of the SF follows what was done in Sect. 4.1 (two parameters fixed). The fit did not succeed for the SF (d), the corresponding time scale was thus rejected. 
P. Favre et al.: AGN variability time scales and the discrete-event model, Online Material p 14
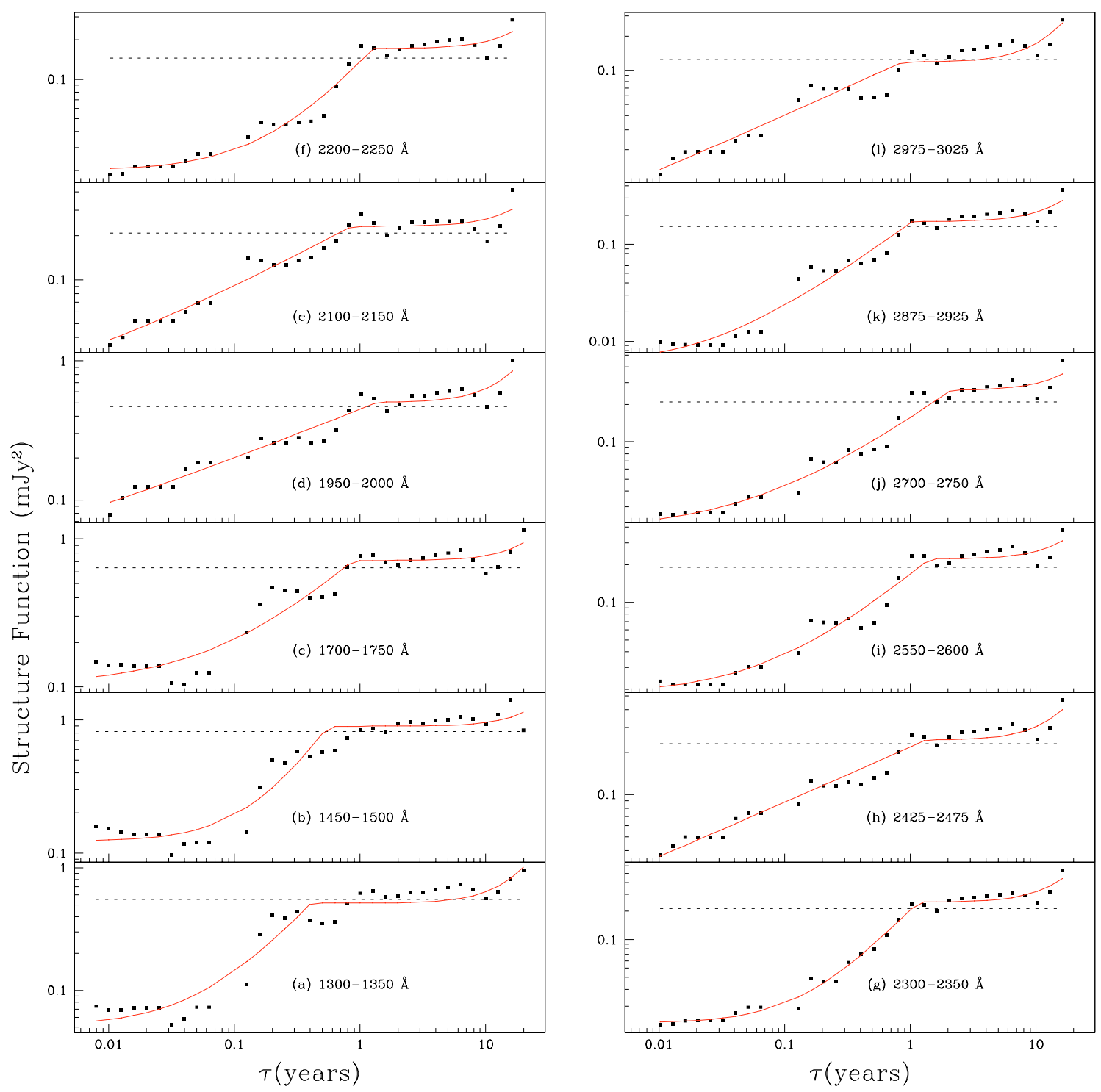

Fig. A.15. NGC 4593, SF for the light curves 1300 to $3000 \AA$ A. 
P. Favre et al.: AGN variability time scales and the discrete-event model, Online Material p 15
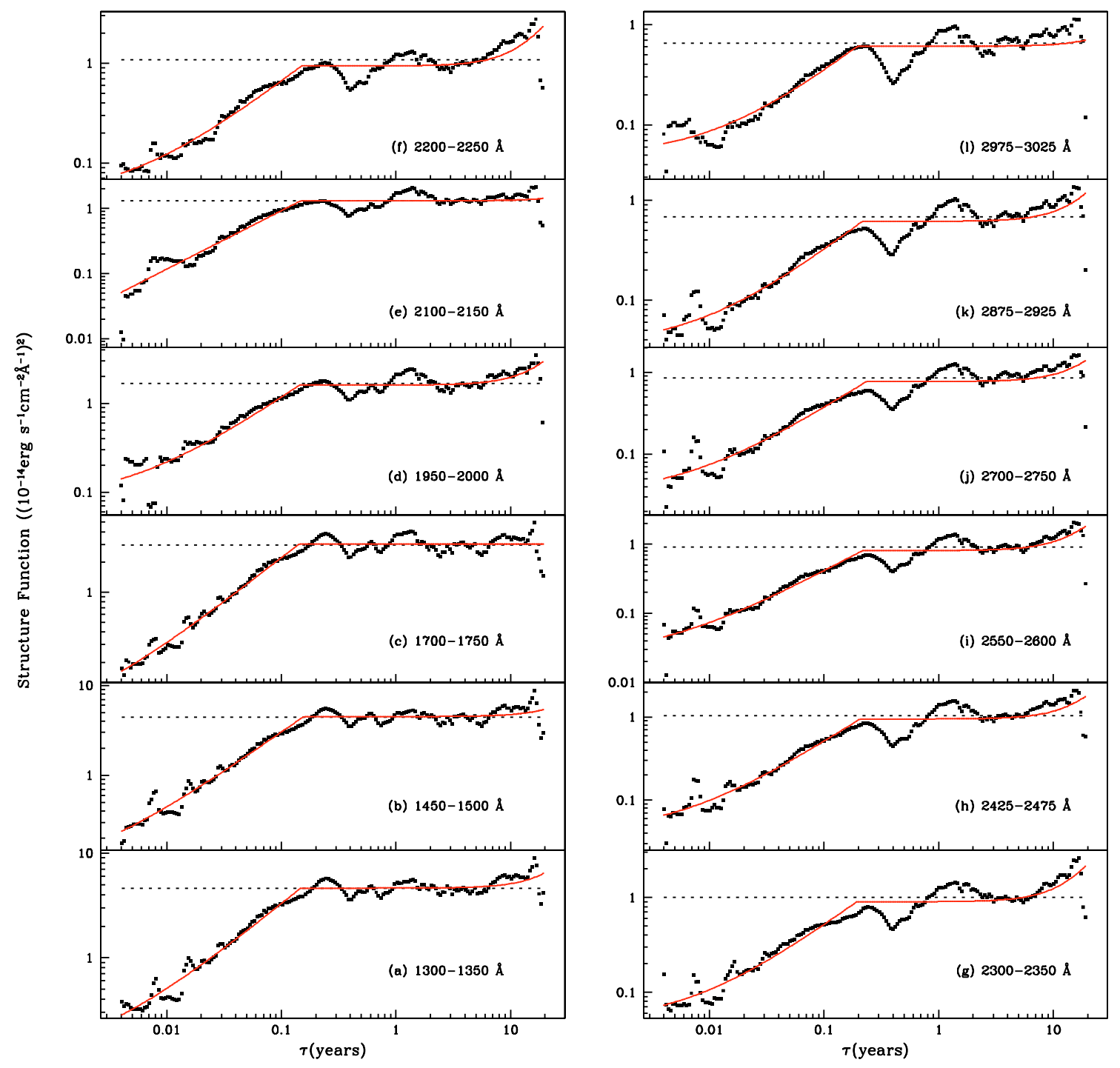

Fig. A.16. NGC 5548, SF for the light curves 1300 to $3000 \AA$. 
P. Favre et al.: AGN variability time scales and the discrete-event model, Online Material $p 16$

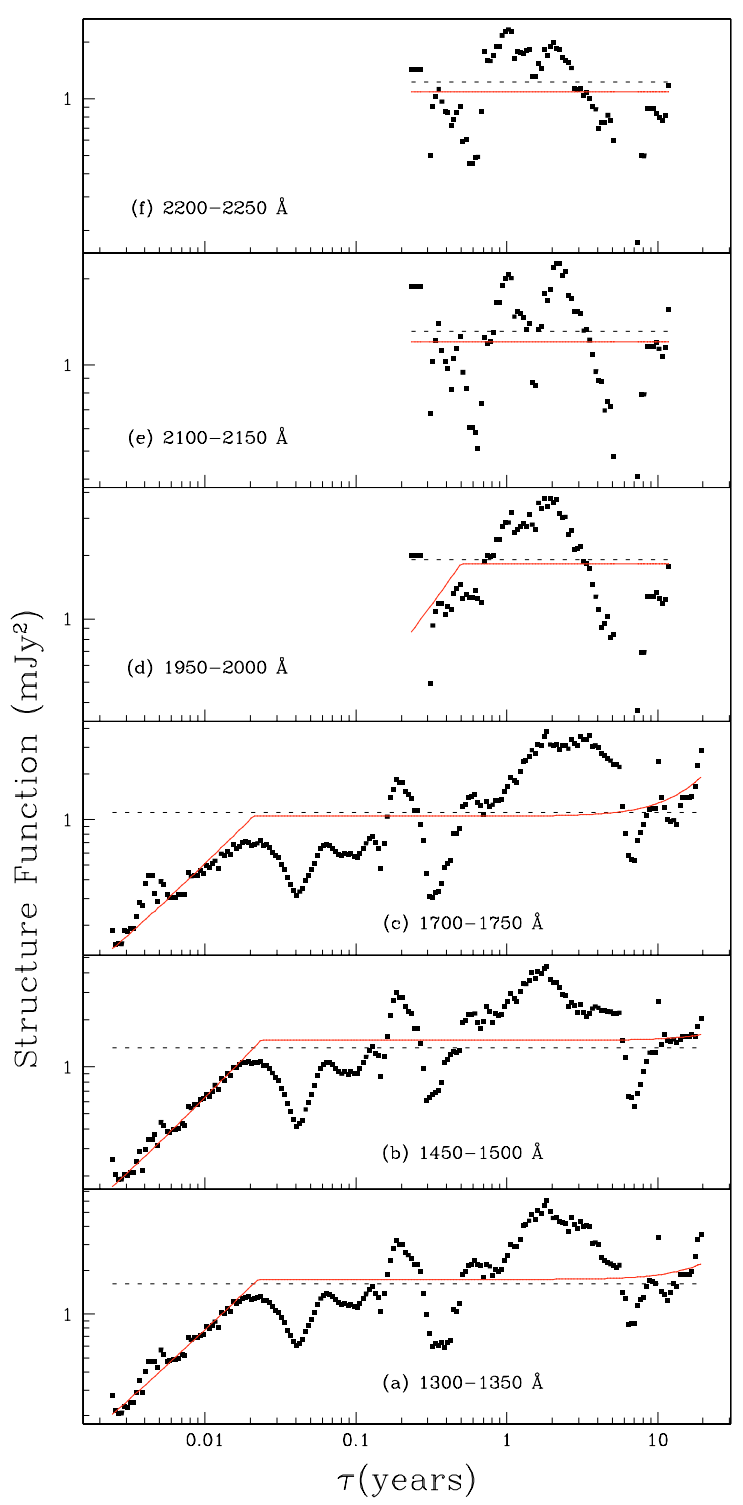

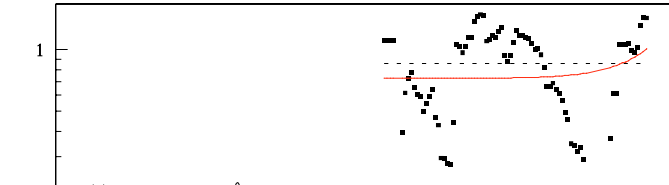

(1) $2975-3025 \AA$

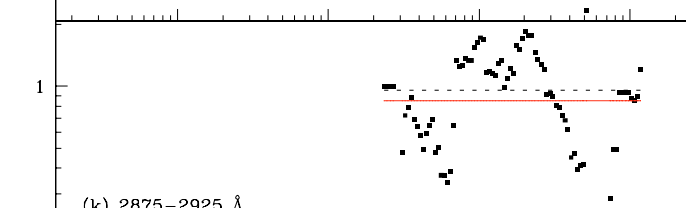

(k) $2875-2925 \AA$

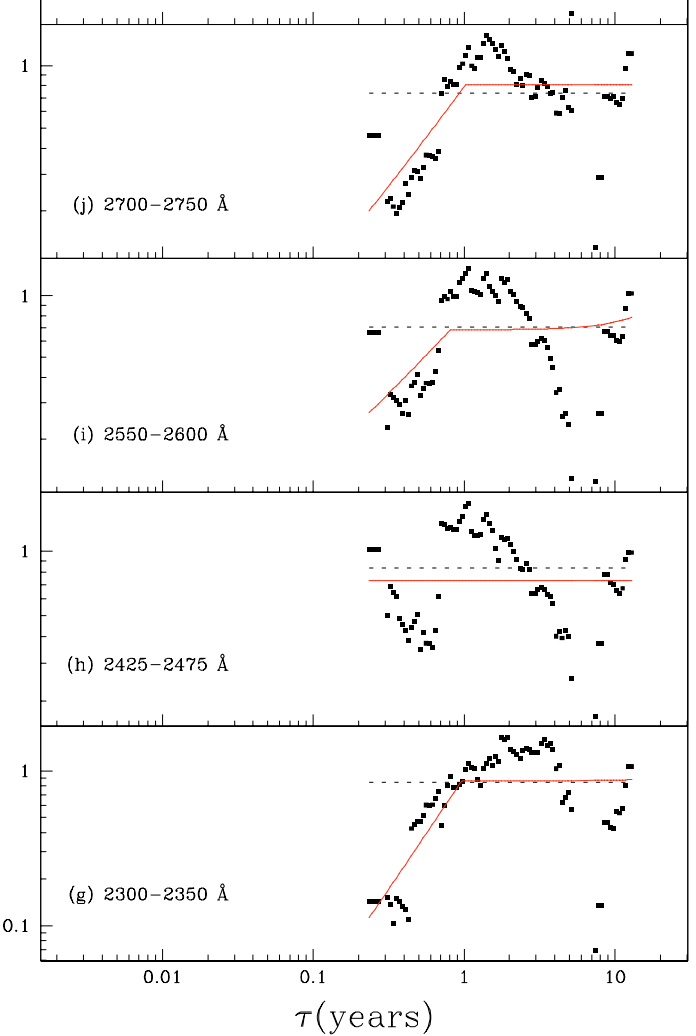

Fig. A.17. NGC 7469, SF for the light curves 1300 to $3000 \AA$ A. The fit of the SF (a)-(c) follows what was done in Sect. 4.1 (two parameters fixed). Only SWP was used for the intensive monitoring made in 1996 (see Fig. A.18). As in NGC 3516, the number of LW epochs is smaller (16) than for the SWP (95). The fit did not succeed for the SF (e), (f), (h), (k), (l). The corresponding time scales were thus rejected. The SF (d), (g), (i), (j) may be interpreted as showing evidence for the presence of a longer timescale in the data, but this result should be taken with caution because of the small number of epochs taken into account. 
P. Favre et al.: AGN variability time scales and the discrete-event model, Online Material p 17

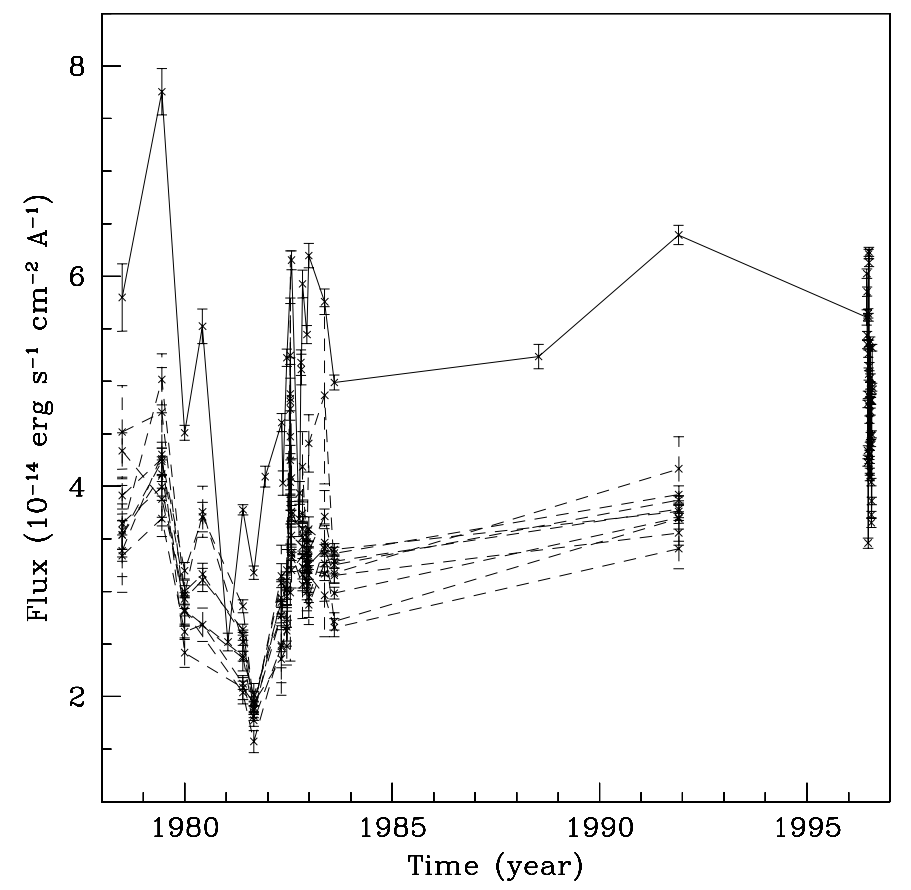

Fig. A.18. NGC 7469, comparison of the LW light curves (dashed lines) with the 1300-1350 A light curve. 
P. Favre et al.: AGN variability time scales and the discrete-event model, Online Material $p 18$
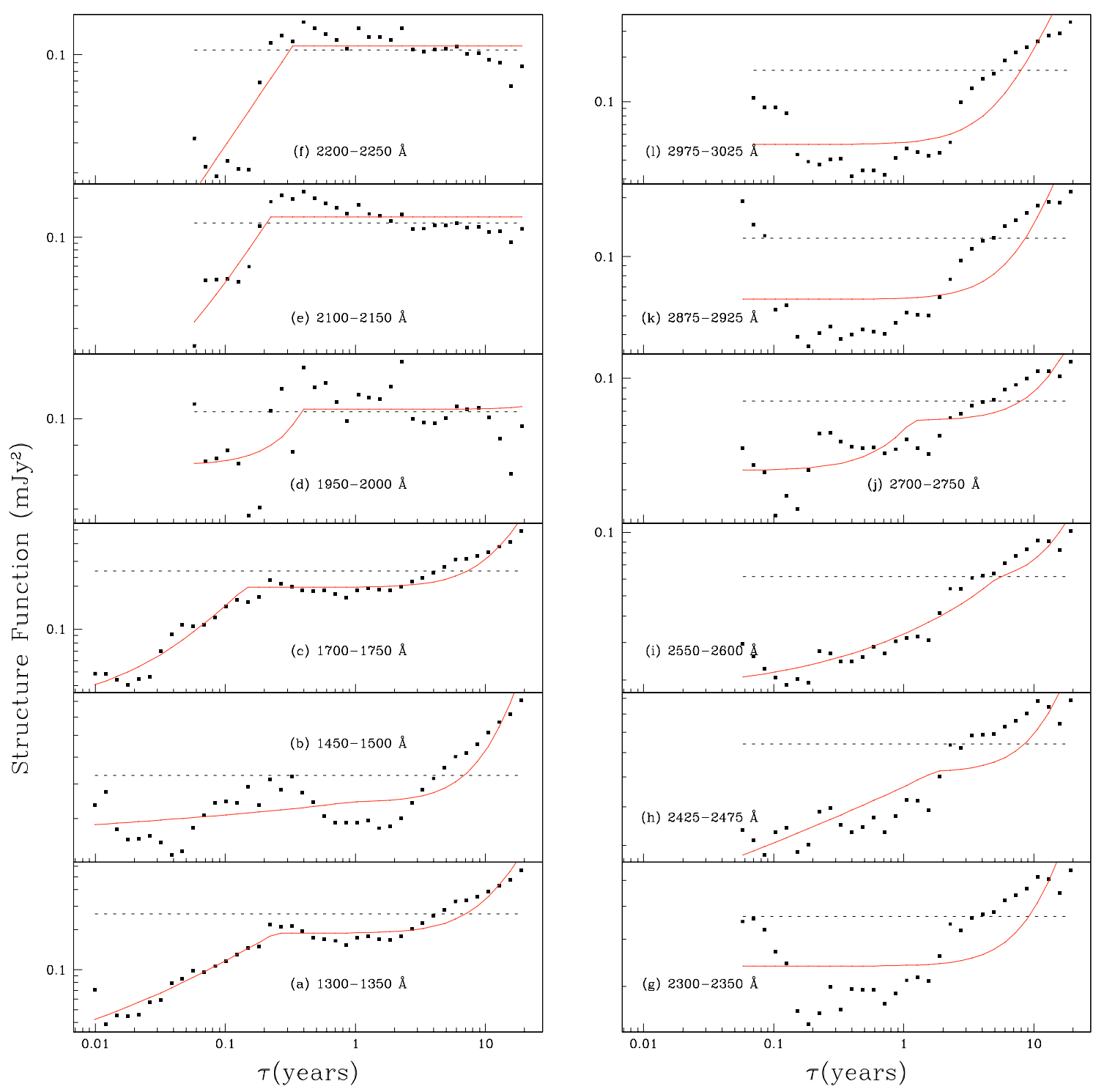

Fig. A.19. 3C 120.0, SF for the light curves 1300 to $3000 \AA$ A. The fit did not succeed for the SF (b), (d), (g)-(l) because of noisy light curves (see Fig. A.20, A.21). The time scales corresponding to these SF are thus rejected. 
P. Favre et al.: AGN variability time scales and the discrete-event model, Online Material $p 19$

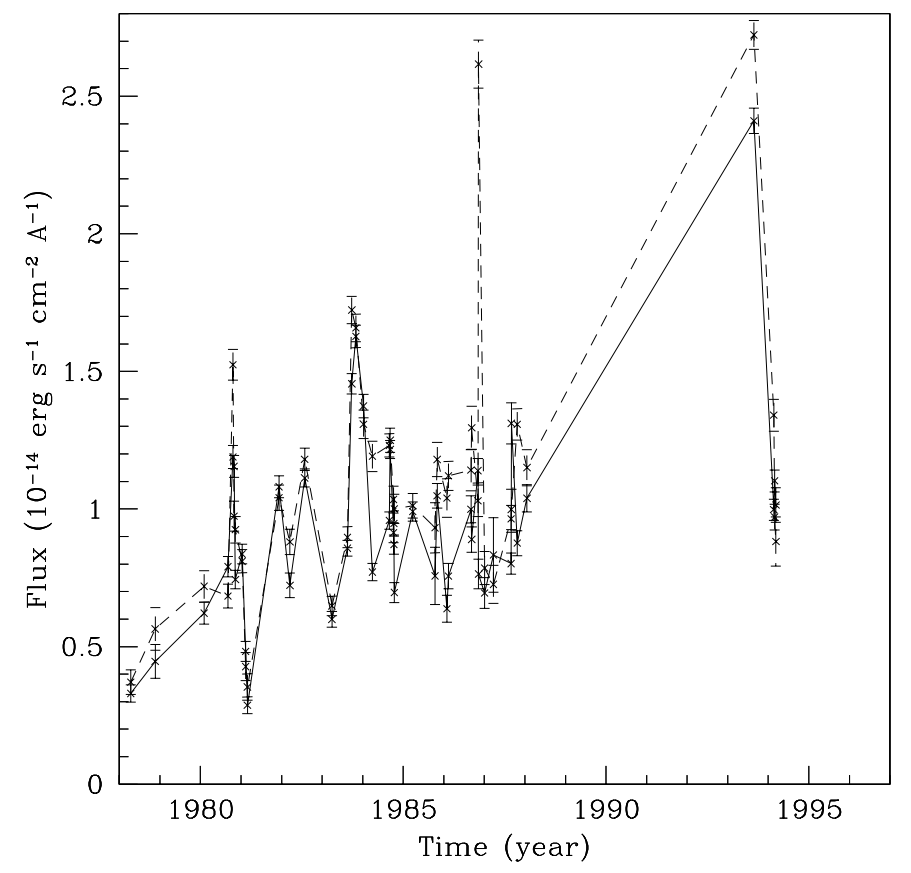

Fig. A.20. 3C 120.0, comparison of the noisy 1450-1500 A light curve (dashed line) with the 1300-1350 Å light curve.

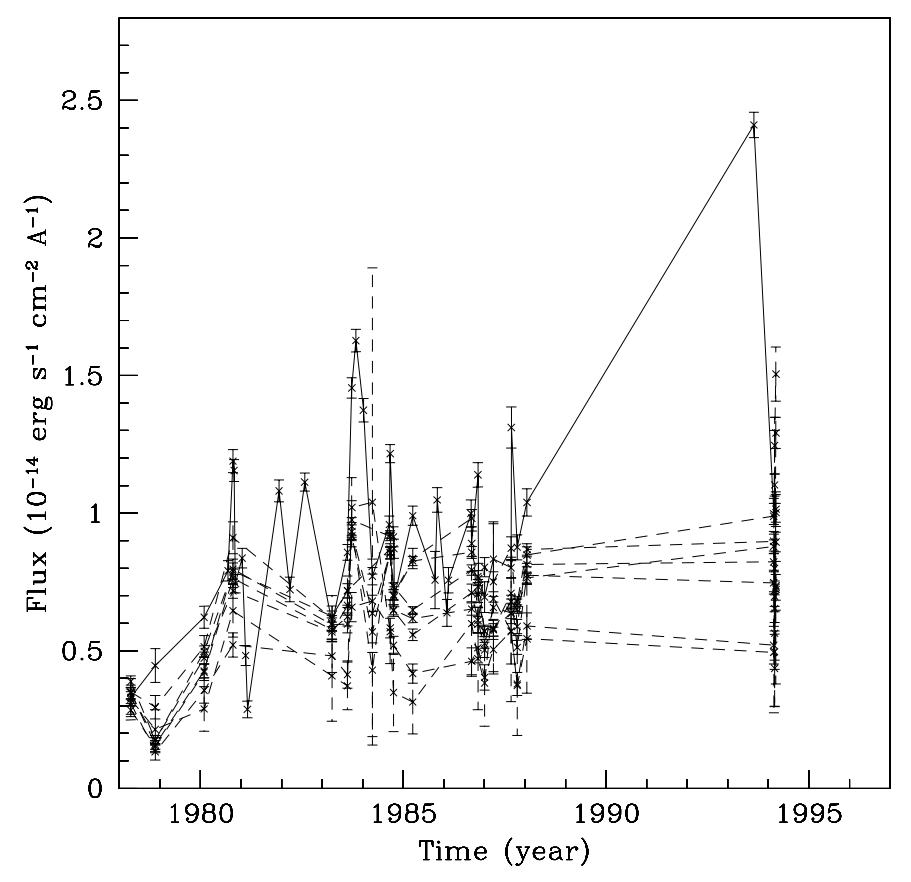

Fig. A.21. 3C 120.0, comparison of the noisy LW (d), (g)-(l) light curves (dashed lines) with the 1300-1350 Å light curve. 
P. Favre et al.: AGN variability time scales and the discrete-event model, Online Material p 20
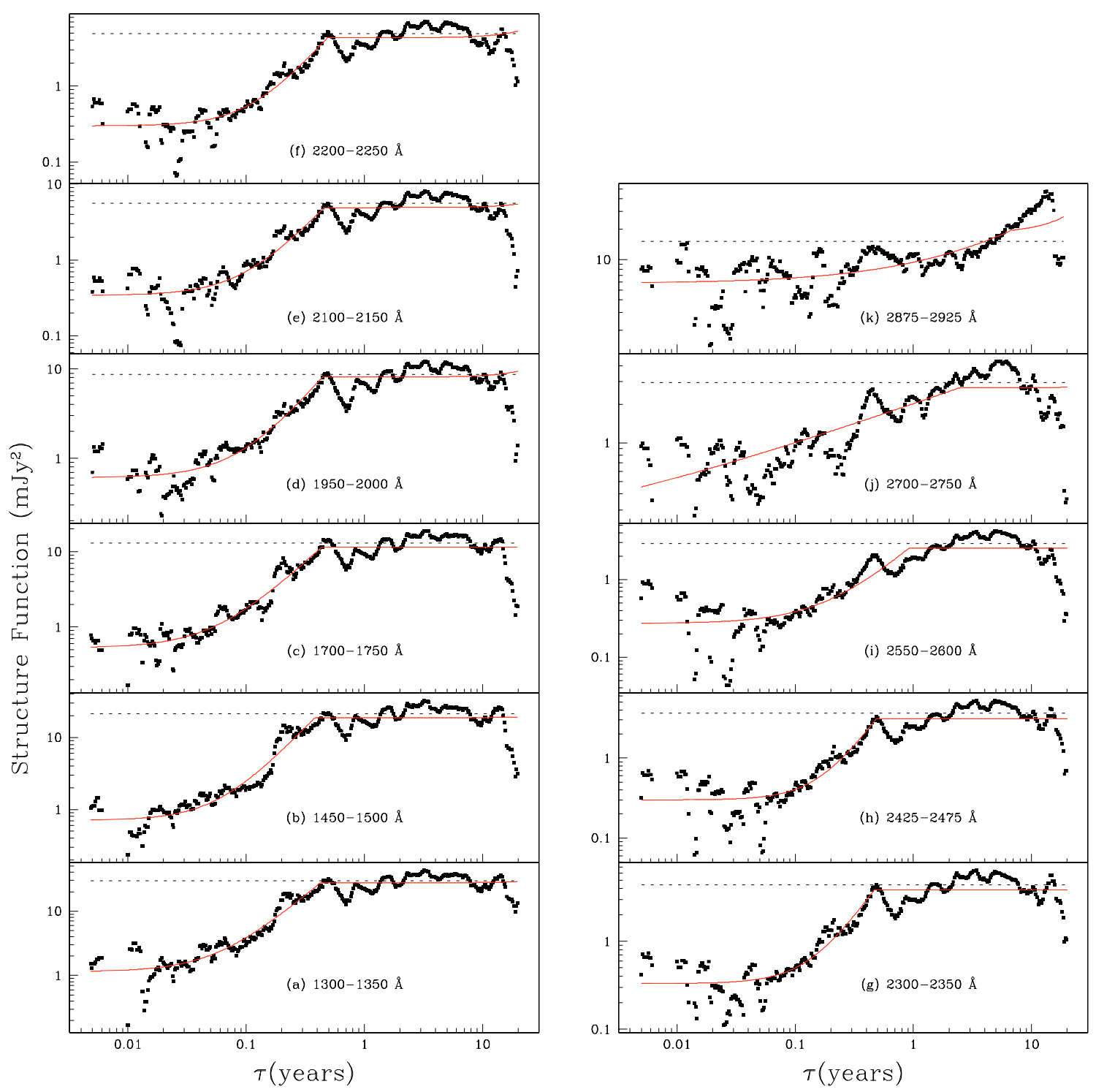

Fig. A.22. 3C 273, SF for the light curves 1300 to $3000 \AA$. The fit did not succeed for the SF (i)-(k) because of noisy light curves (see Fig. A.23-A.25). The time scales corresponding to these SF are thus rejected. 
P. Favre et al.: AGN variability time scales and the discrete-event model, Online Material p 21

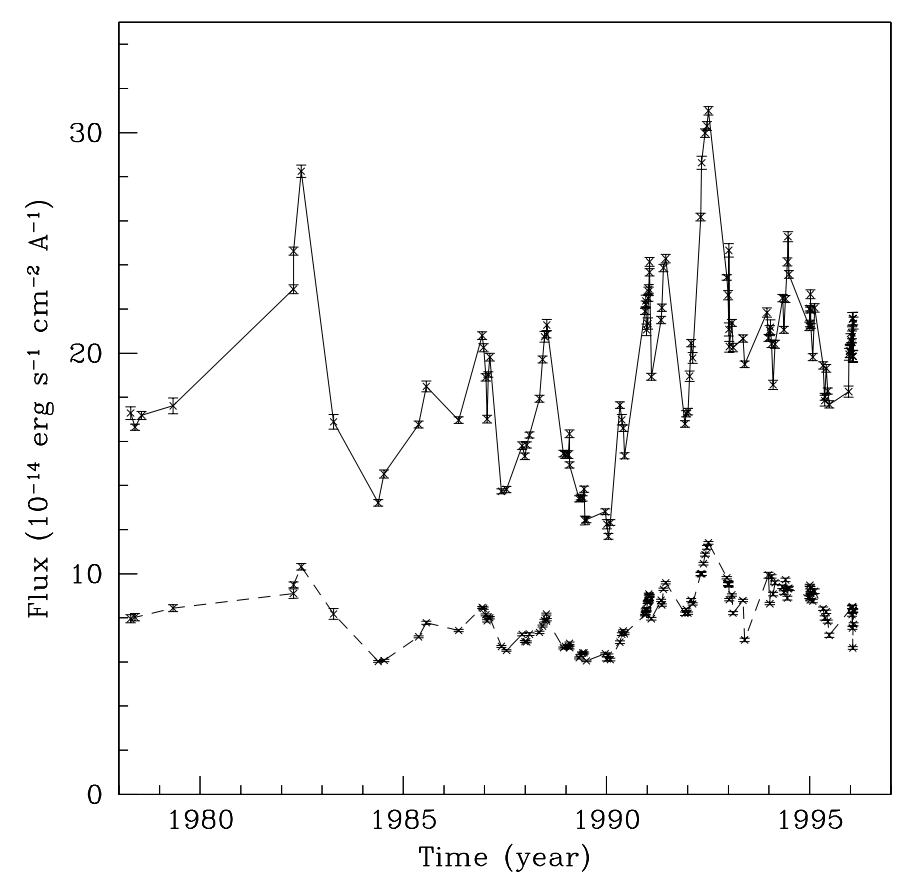

Fig. A.23. 3C 273, comparison of the noisy 2550-2600 ̊ light curve (dashed line) with the 1300-1350 Å light curve.

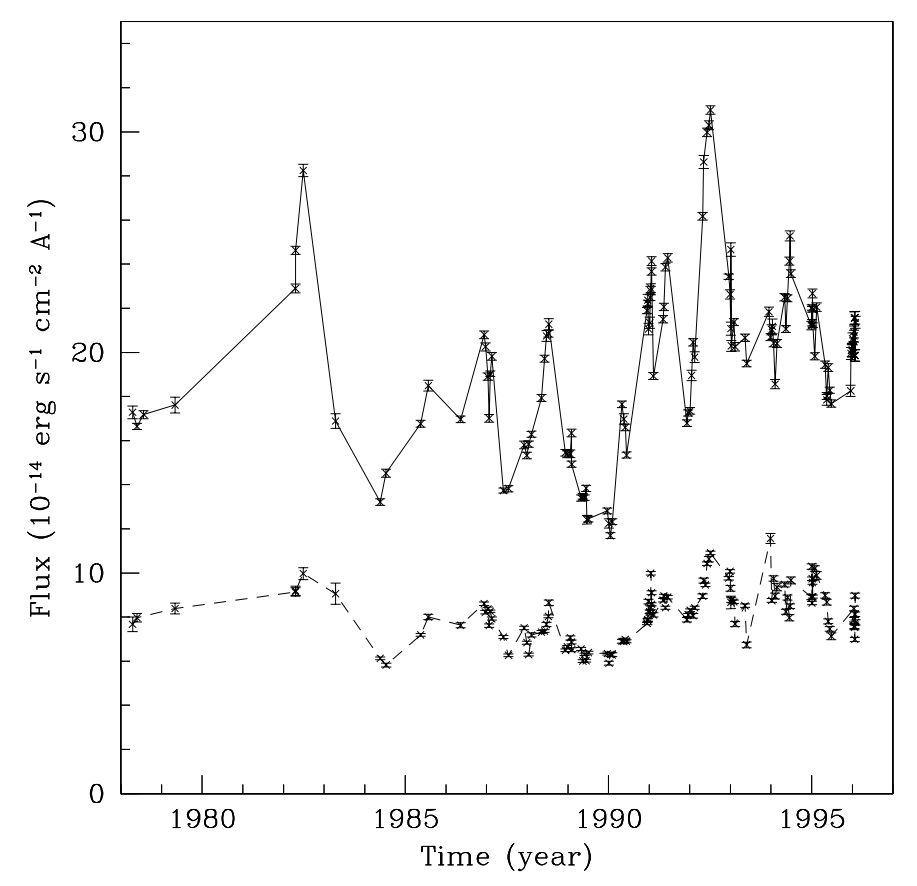

Fig. A.24. 3C 273, comparison of the noisy $2700-2750 \AA$ light curve (dashed line) with the 1300-1350 A light curve.

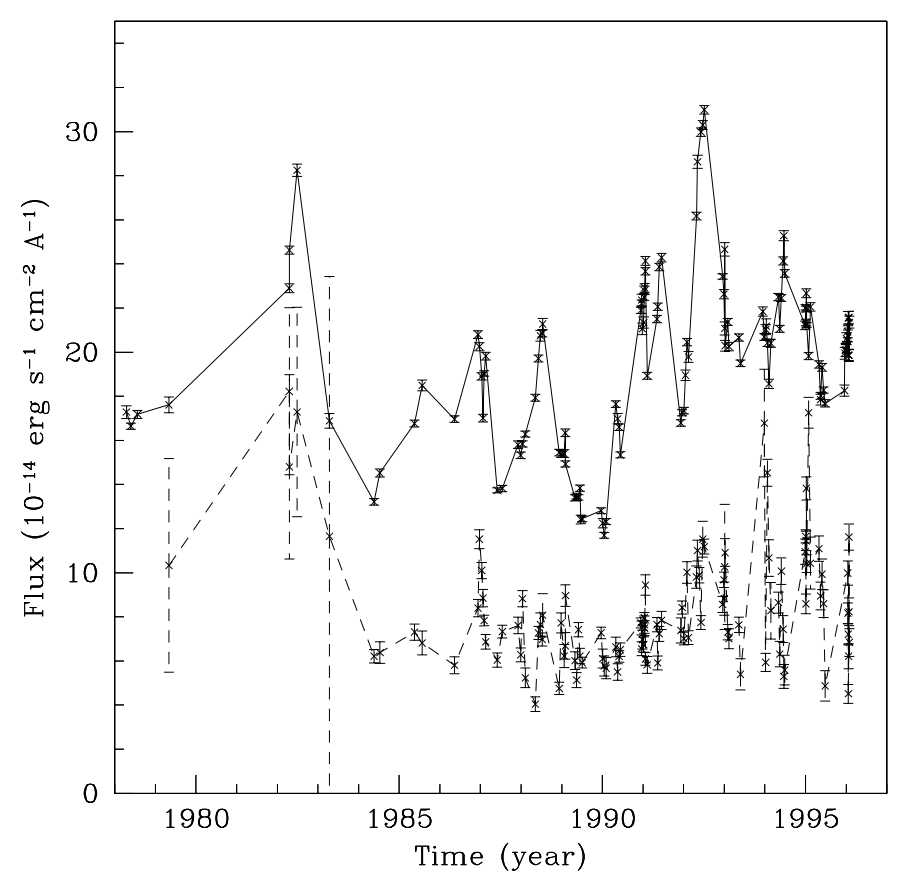

Fig. A.25. 3C 273, comparison of the noisy 2875-2925 ̊ light curve (dashed line) with the 1300-1350 A light curve. 
P. Favre et al.: AGN variability time scales and the discrete-event model, Online Material p 22
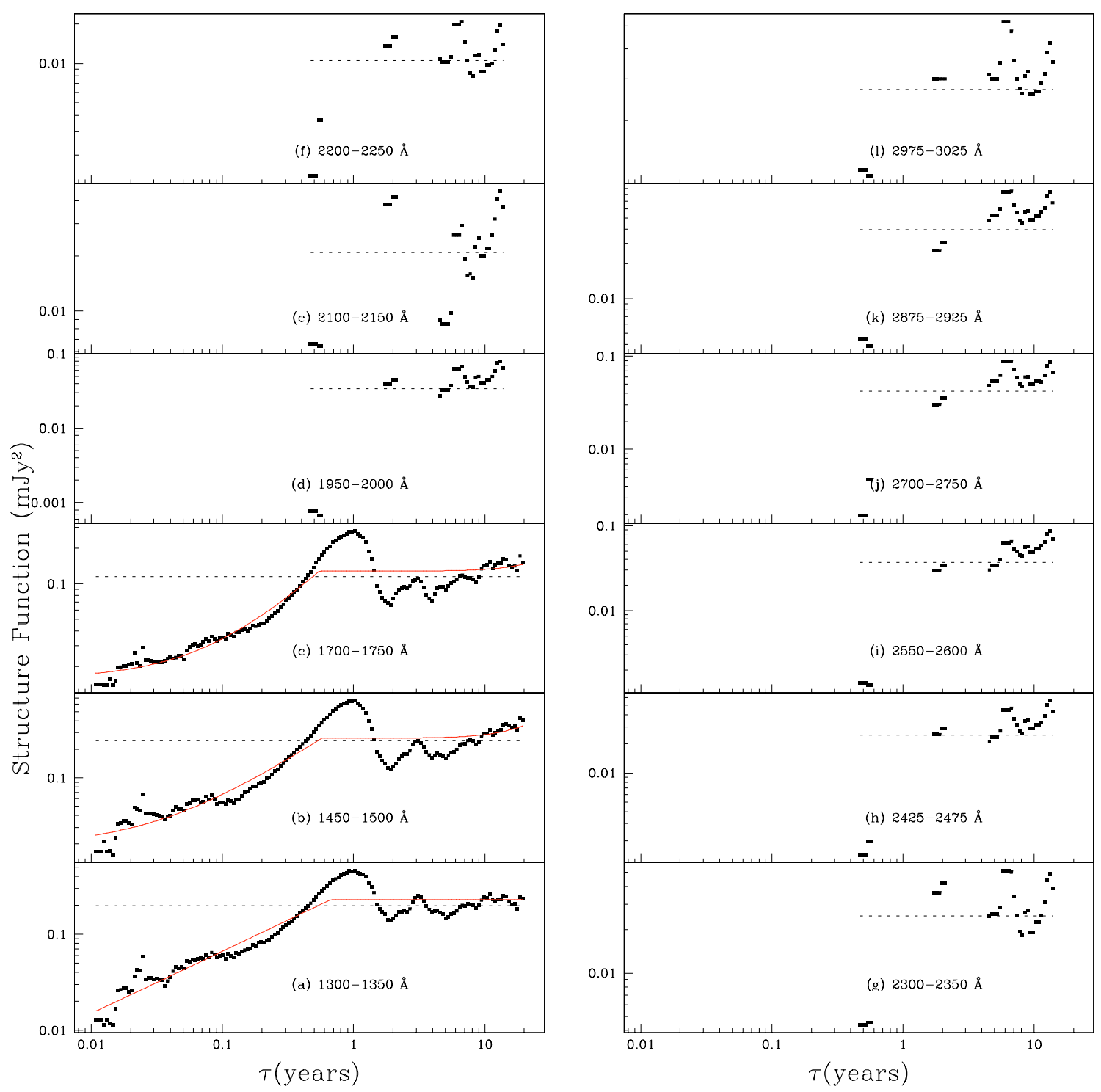

Fig. A.26. 3C 390.3, SF for the light curves 1300 to $3000 \AA$ A. Only SWP was used for the intensive monitoring made in $1995-1996$ (see Fig. A.27). The number of LW epochs is too small to build measurable SF. The fit did not succeed and no time scales could be deduced in the range 1950 to $2975 \AA$ for this object. 
P. Favre et al.: AGN variability time scales and the discrete-event model, Online Material p 23

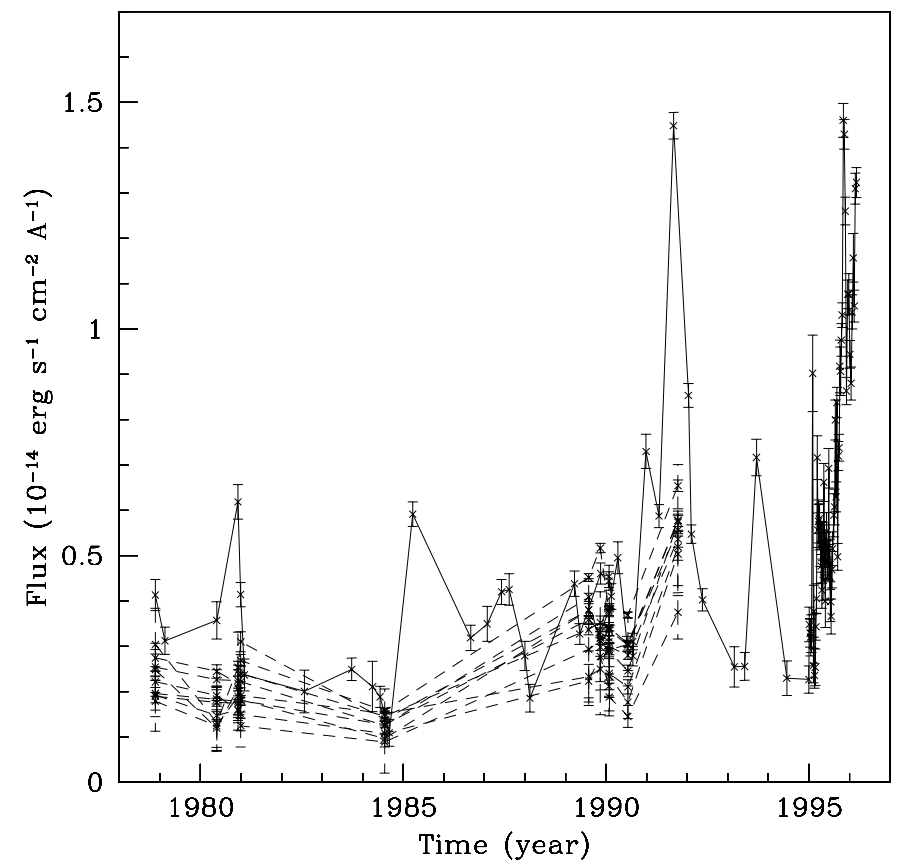

Fig. A.27. 3C 390.3, comparison of the LW light curves (dashed lines) with the 1300-1350 Å light curve. 
P. Favre et al.: AGN variability time scales and the discrete-event model, Online Material p 24
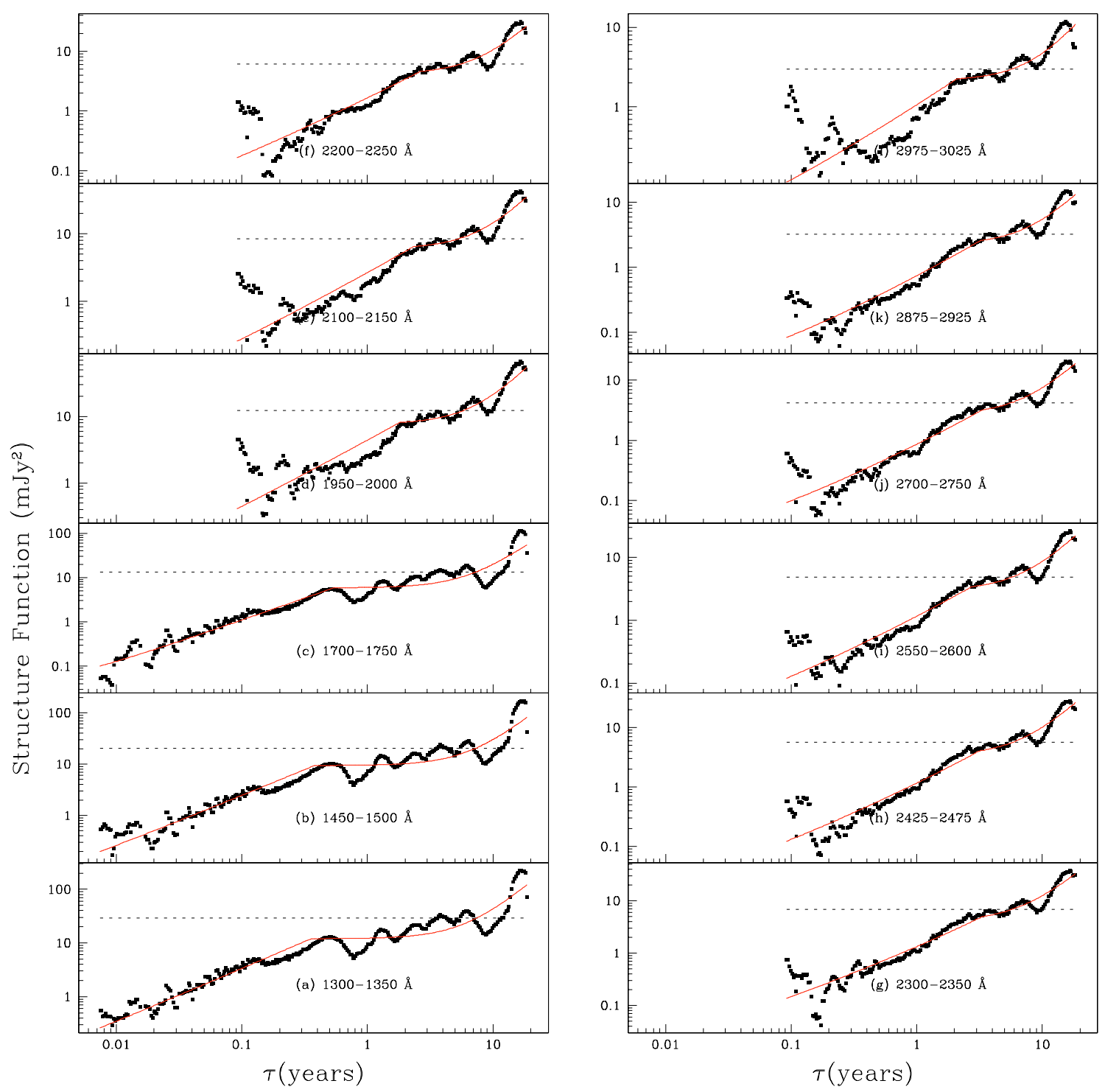

Fig. A.28. Fairall 9, SF for the light curves 1300 to $3000 \AA$ A. The fit of the SF (a)-(c) follows what was done in Sect. 4.1 (two parameters fixed). The number of epochs between SWP and LW is very uneven (139 for the SWP and 63 for the LW). The SF (d)-(1) may be interpreted as showing evidence for the presence of a longer timescale in the data. 
P. Favre et al.: AGN variability time scales and the discrete-event model, Online Material p 25
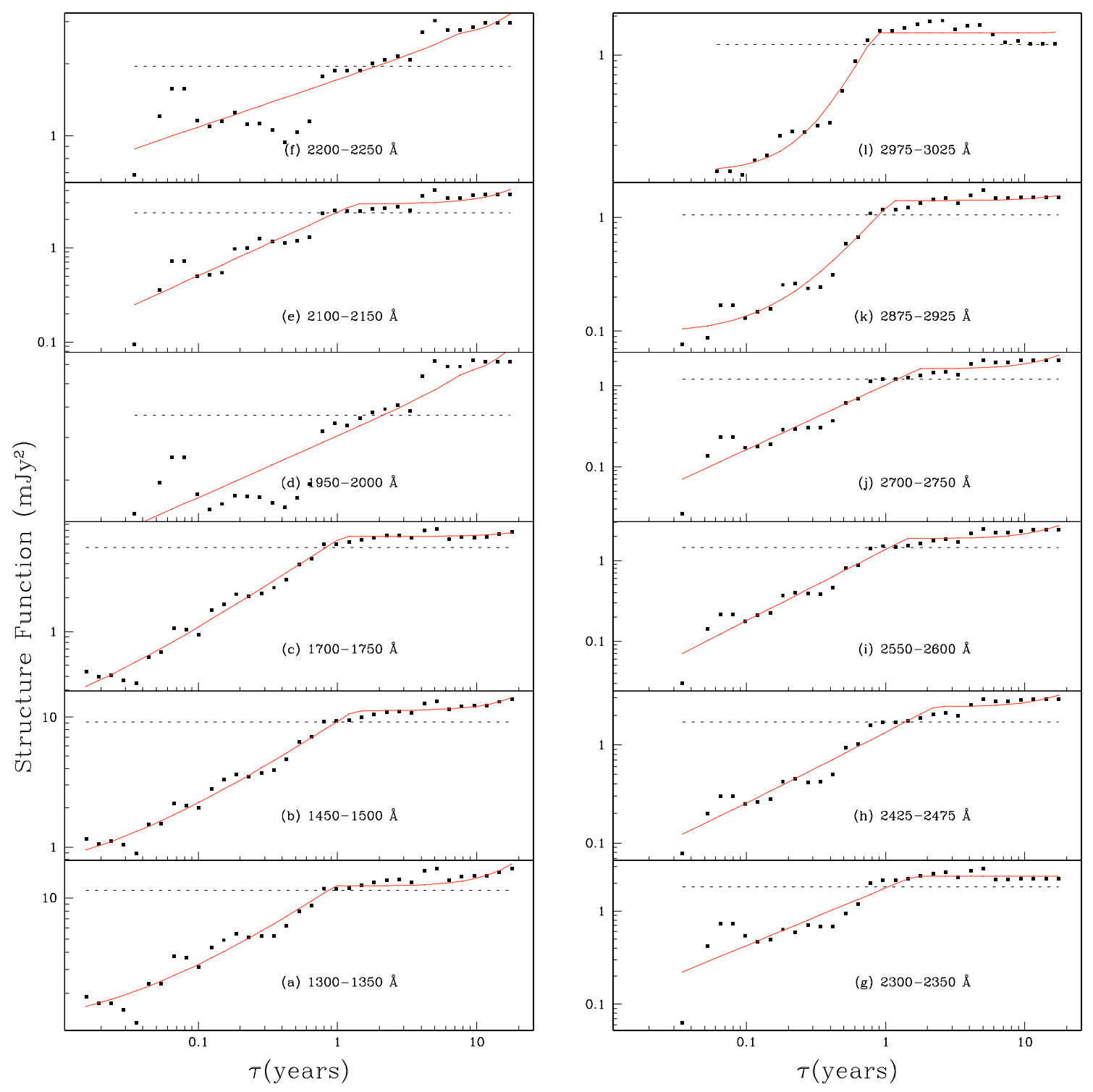

Fig. A.29. ESO 141-55, SF for the light curves 1300 to $3000 \AA$ A. The fit did not succeed for the SF (d), (f) due to noisy light curves (see Fig. A.30); the corresponding time scales were thus rejected. 
P. Favre et al.: AGN variability time scales and the discrete-event model, Online Material p 26

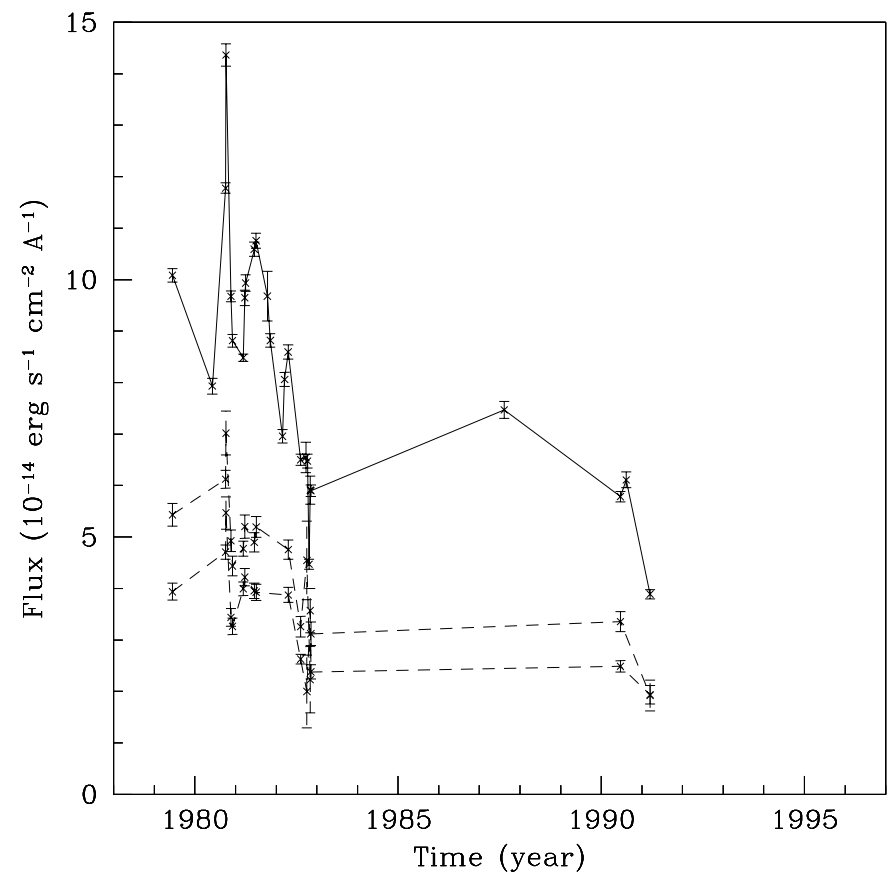

Fig. A.30. ESO 141-55, comparison of the noisy 1950-2000 $\AA$ and 2200-2250 A light curves (dashed lines) with the 1300-1350 ̊ light curve. 